\title{
Blowing up solutions for an elliptic Neumann problem with sub- or supercritical nonlinearity. Part II: $N \geqslant 4$ 光
}

\section{Solutions concentrées pour un problème elliptique de Neumann avec non-linéarité sous- ou sur-critique. II: $N \geqslant 4$}

\author{
Olivier Rey ${ }^{\mathrm{a}, *}$, Juncheng Wei ${ }^{\mathrm{b}}$ \\ ${ }^{a}$ Centre de Mathématiques de l'Ecole Polytechnique, 91128 Palaiseau cedex, France \\ ${ }^{\mathrm{b}}$ Department of Mathematics, Chinese University of Hong Kong, Shatin, Hong Kong
}

Received 15 April 2004; accepted 15 July 2004

Available online 7 April 2005

\begin{abstract}
We consider the sub- or supercritical Neumann elliptic problem $-\Delta u+\mu u=u^{\frac{N+2}{N-2}+\varepsilon}, u>0$ in $\Omega ; \frac{\partial u}{\partial n}=0$ on $\partial \Omega, \Omega$ being a smooth bounded domain in $\mathbb{R}^{N}, N \geqslant 4, \mu>0$ and $\varepsilon \neq 0$ a small number. We show that for $\varepsilon>0$, there always exists a solution to the slightly supercritical problem, which blows up at the most curved part of the boundary as $\varepsilon$ goes to zero. On the other hand, for $\varepsilon<0$, assuming that the domain is not convex, there also exists a solution to the slightly subcritical problem, which blows up at the least curved part of the domain.
\end{abstract}

(C) 2005 L'Association Publications de l'Institut Henri Poincaré. Published by Elsevier B.V. All rights reserved

\section{Résumé}

$\Omega$ étant un domaine borné régulier de $\mathbb{R}^{N}, N \geqslant 4$, on considère le problème elliptique de Neumann $-\Delta u+\mu u=u^{\frac{N+2}{N-2}+\varepsilon}$, $u>0$ dans $\Omega ; \frac{\partial u}{\partial n}=0$ sur $\partial \Omega$, où $\mu>0$ est un paramètre fixé. On montre que pour $\varepsilon>0$ assez petit, le problème admet une solution non-constante, qui se concentre quand $\varepsilon$ tend vers zéro en un point de la frontière où la courbure moyenne est maximum. En supposant que le domaine n'est pas convexe, on montre aussi, pour $\varepsilon<0$ assez proche de zéro, l'existence d'une solution non-constante, qui se concentre quand $\varepsilon$ tend vers zéro en un point de la frontière où la courbure moyenne est minimum. (C) 2005 L'Association Publications de l'Institut Henri Poincaré. Published by Elsevier B.V. All rights reserved

\footnotetext{
Research supported in part by an Earmarked Grant from RGC of HK.

* Corresponding author.

E-mail addresses: rey@math.polytechnique.fr (O. Rey), wei@math.cuhk.edu.hk (J. Wei).
} 


\section{Introduction}

In this paper we consider the nonlinear Neumann elliptic problem

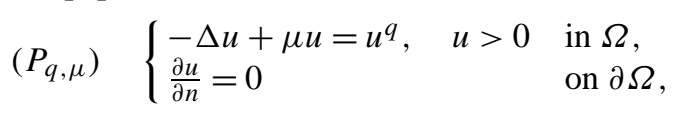

where $1<q<+\infty, \mu>0$ and $\Omega$ is a smooth and bounded domain in $\mathbb{R}^{N}, N \geqslant 4$.

Eq. $\left(P_{q, \mu}\right)$ arises in many branches of the applied sciences. For example, it can be viewed as a steady-state equation for the shadow system of the Gierer-Meinhardt system in biological pattern formation [12,27], or for parabolic equations in chemotaxis, e.g. Keller-Segel model [24].

When $q$ is subcritical, i.e. $q<\frac{N+2}{N-2}$, Lin, Ni and Takagi proved that the only solution, for small $\mu$, is the constant one, whereas nonconstant solutions appear for large $\mu$ [24] which blow up, as $\mu$ goes to infinity, at one or several points. The least energy solution blows up at a boundary point which maximizes the mean curvature of the frontier $[29,30]$. Higher energy solutions exist which blow up at one or several points, located on the boundary $[8,13,22$, $42,18]$, in the interior of the domain $[5,7,10,11,15,20,40,43]$, or some of them on the boundary and others in the interior [17]. (A good review can be found in [27].) In the critical case, i.e. $q=5$, Zhu [44] proved that, for convex domains, the only solution is the constant one for small $\mu$ (see also [41]). For large $\mu$, nonconstant solutions exist $[1,35]$. As in the subcritical case the least energy solution blows up, as $\mu$ goes to infinity, at a unique point which maximizes the mean curvature of the boundary $[3,28]$. Higher energy solutions have also been exhibited, blowing up at one $[2,36,32,14]$ or several boundary points $[26,37,38,16]$. The question of interior blow-up is still open. However, in contrast with the subcritical situation, at least one blow-up point has to lie on the boundary [33].

Very few is known about the supercritical case, save the uniqueness of the radial solution on a ball for small $\mu$ [23]. In [27], Ni raised the following conjecture.

Conjecture. For any exponent $q>1$, and $\mu$ large, there always exists a nonconstant solution to $\left(P_{q, \mu}\right)$.

Our aim, in this paper, is to continue our study [34] on the problem for fixed $\mu$, when the exponent $q$ is close to the critical one, i.e. $q=\frac{N+2}{N-2}+\varepsilon$ and $\varepsilon$ is a small nonzero number. Whereas the previous results, concerned with peaked solutions, always assume that $\mu$ goes to infinity, we are going to prove that a single interior or boundary peak solution may exist for fixed $\mu$, provided that $q$ is close enough to the critical exponent. In [34], we showed that for $N=3$, a single interior bubble solution exists for finite $\mu$, as $\varepsilon \rightarrow 0$. In this paper, we establish the existence of a single boundary bubble for any finite $\mu$ and for any smooth bounded domain $\Omega \subset \mathbb{R}^{N}, N \geqslant 4$, provided that $\varepsilon>0$ is sufficiently small.

Let $H(a)$ denote the boundary mean curvature function at $a \in \partial \Omega$. The following result partially answers Ni's conjecture:

Theorem 1.1. Suppose that $N \geqslant 4$. Then $\left(P_{\frac{N+2}{N-2}+\varepsilon, \mu}\right)$ has a nontrivial solution, for $\varepsilon>0$ close enough to zero, which blows up as $\varepsilon$ goes to zero at a point $a \in \partial \Omega$, such that $H(a)=\max _{P \in \partial \Omega} H(P)$.

In the case of $\varepsilon<0$, i.e. slightly subcritical case, we then have the following theorem.

Theorem 1.2. Assume that $N \geqslant 4$ and $\Omega$ is not convex. Then $\left(P_{\frac{N+2}{N-2}+\varepsilon, \mu}\right)$ has a nontrivial solution, for $\varepsilon<0$ close enough to zero, which blows up as $\varepsilon$ goes to zero at a point $a \in \partial \Omega$, such that $H(a)=\min _{P \in \partial \Omega} H(P)$.

Remark. Theorem 1.2 agrees with the following result of Gui and Lin: in [14], it is proved that if there exists a sequence of single boundary blowing up solutions $u_{\varepsilon_{i}}$ to $P_{\frac{N+2}{N-2}+\varepsilon_{i}, \mu}$ with $\varepsilon_{i} \leqslant 0$, then necessarily, $u_{\varepsilon_{i}}$ blows up at a boundary point $a \in \partial \Omega$ such that $H(a) \leqslant 0$ and $a$ is a critical point of $H$. Here we have established a partial converse to [14]. 
A similar slightly supercritical Dirichlet problem

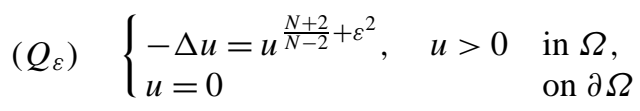

has been studied in [9], where the existence of solutions with two bubbles in domains with a small hole is established, provided that $\varepsilon$ is small. It is interesting to note that, here, and also in [34], we have no condition on the domain, in the slightly supercritical Neumann case.

The scheme of the proof is similar to [34] (see also [9]). However, we use a different framework - i.e. weighted Sobolev spaces - to treat the case $N \geqslant 4$. In the next section, we define a two-parameters set of approximate solutions to the problem, and we look for a true solution in a neighborhood of this set. Considering in Section 3 the linearized problem at an approximate solution, and inverting it in suitable functional spaces, the problem reduces to a finite dimensional one, which is solved in Section 4. Some useful facts and computations are collected in Appendix.

\section{Some preliminaries}

\subsection{Approximate solutions and rescaling}

For sake of simplicity, we consider in the following the supercritical case, i.e. we assume that $\varepsilon>0$. The subcritical case may be treated exactly in the same way. For normalization reasons, we consider throughout the paper the equation

$$
-\Delta u+\mu u=\alpha_{N} u^{\frac{N+2}{N-2}+\varepsilon}, \quad u>0,
$$

instead of the original one, where $\alpha_{N}=N(N-2)$. The solutions are identical, up to the multiplicative constant $\left(\alpha_{N}\right)^{-\frac{N-2}{4+(N-2) \varepsilon}}$. We recall that, according to [6], the functions

$$
U_{\lambda, a}(x)=\frac{\lambda^{\frac{N-2}{2}}}{\left(1+\lambda^{2}|x-a|^{2}\right)^{\frac{N-2}{2}}}, \quad \lambda>0, a \in \mathbb{R}^{N},
$$

are the only solutions to the problem

$$
-\Delta u=\alpha_{N} u^{\frac{N+2}{N-2}}, \quad u>0 \quad \text { in } \mathbb{R}^{N} .
$$

As $a \in \partial \Omega$ and $\lambda$ goes to infinity, these functions provide us with approximate solutions to the problem that we are interested in. However, in view of the additional linear term $\mu u$ which occurs in $\left(P_{\frac{N+2}{N-2}+\varepsilon, \mu}\right)$, the approximation needs to be improved.

Integral estimates (see Appendix) suggest to make the additional a priori assumption that $\lambda$ behaves as $1 / \varepsilon$ as $\varepsilon$ goes to zero. Namely, we set

$$
\lambda=\frac{1}{\Lambda \varepsilon}, \quad \frac{1}{\delta^{\prime}}<\Lambda<\delta^{\prime}
$$

with $\delta^{\prime}$ some strictly positive number. Now, fix $a \in \partial \Omega$. We define $V_{\Lambda, a, \mu, \varepsilon}=V$ satisfying

$$
\begin{cases}-\Delta V+\mu V=\alpha_{N} U_{\frac{1}{\Lambda \varepsilon}, a}^{\frac{N+2}{N-2}} & \text { in } \Omega, \\ \frac{\partial V}{\partial n}=0 & \text { on } \partial \Omega .\end{cases}
$$

The $V_{\Lambda, a, \mu, \varepsilon}$ 's are the suitable approximate solutions in the neighborhood of which we shall find a true solution to the problem. In order to make further computations easier, we proceed to a rescaling. We set

$$
\Omega_{\varepsilon}=\frac{\Omega}{\varepsilon}
$$


and define in $\Omega_{\varepsilon}$ the functions

$$
W_{\Lambda, \xi, \mu, \varepsilon}(x)=\varepsilon^{\frac{N-2}{2}} V_{\Lambda, a, \mu, \varepsilon}(\varepsilon x), \quad \xi=\frac{a}{\varepsilon} .
$$

$W_{\Lambda, \xi, \mu, \varepsilon}=W$ satisfies

$$
\begin{cases}-\Delta W+\mu \varepsilon^{2} W=\alpha_{N} U_{\frac{1}{\Lambda}, \xi}^{\frac{N+2}{N-2}} & \text { in } \Omega_{\varepsilon} \\ \frac{\partial W}{\partial n}=0 & \text { on } \partial \Omega_{\varepsilon}\end{cases}
$$

and, since $U_{\frac{1}{\Lambda}, \xi} \geqslant C \epsilon^{N-2}$ and $\Delta W \geqslant 0$ at a minimum point of $W$ in the closure of $\Omega$

$$
W \geqslant C \epsilon^{N} \text { in } \bar{\Omega} \text {. }
$$

Another fact that we shall use later is the following: observe that $\partial_{\Lambda} W$ satisfies

$$
\begin{cases}-\Delta\left(\partial_{\Lambda} W\right)+\mu \varepsilon^{2} \partial_{\Lambda} W=\alpha_{N} \partial_{\Lambda}\left(U_{\frac{1}{\Lambda}, \xi}^{\left.\frac{N+2}{N-2}\right)}\right. & \text { in } \Omega_{\varepsilon} \\ \frac{\partial\left(\partial_{\Lambda} W\right)}{\partial n}=0 & \text { on } \partial \Omega_{\varepsilon}\end{cases}
$$

Since $\left|\partial_{\Lambda}\left(U_{\frac{1}{\Lambda}, \xi}^{\frac{N+2}{N-2}}\right)\right| \leqslant C U_{\frac{1}{\Lambda}, \xi}^{\frac{N+2}{N-2}}$, by comparison principle we obtain

$$
\left|\partial_{\Lambda} W\right| \leqslant C W \text {. }
$$

The same holds for $\partial_{\xi} W$ instead of $\partial_{\Lambda} W$.

Finding a solution to $\left(P_{\frac{N+2}{N-2}+\varepsilon, \mu}\right)$ in a neighborhood of the functions $V_{\Lambda, a, \mu, \varepsilon}$ is equivalent, through the following rescaling

$$
u(x) \rightarrow \varepsilon^{-\frac{2(N-2)}{4+(N-2) \varepsilon}} u\left(\frac{x}{\varepsilon}\right)
$$

to solving the problem

$$
\left(P_{\frac{N+2}{N-2}+\varepsilon, \mu}^{\prime}\right) \quad \begin{cases}-\Delta u+\mu \varepsilon^{2} u=\alpha_{N} u^{\frac{N+2}{N-2}+\varepsilon}, \quad u>0 & \text { in } \Omega_{\varepsilon}, \\ \frac{\partial u}{\partial n}=0 & \text { on } \partial \Omega_{\varepsilon}\end{cases}
$$

in a neighborhood of the functions $W_{\Lambda, \xi, \mu, \varepsilon}$. (From now on, we shall work with $\left(P_{\frac{N+2}{N-2}+\varepsilon, \mu}^{\prime}\right)$.) For that purpose, we have to use some local inversion procedure. Namely, we are going to look for a solution to $\left(P_{\varepsilon, \mu}^{\prime}\right)$ writing as

$$
w=W_{\Lambda, \xi, \mu, \varepsilon}+\omega
$$

with $\omega$ small and orthogonal at $W_{\Lambda, \xi, \mu, \varepsilon}$, in a suitable sense, to the manifold

$$
M=\left\{W_{\Lambda, \xi, \mu, \varepsilon}, \Lambda \text { satisfying }(2.3), \xi \in \partial \Omega_{\varepsilon}\right\} .
$$

The general strategy consists in finding first, using an inversion procedure, a smooth map $(\Lambda, \xi) \mapsto \omega(\Lambda, \xi)$ such that $W_{\Lambda, \xi, \mu, \varepsilon}+\omega(\Lambda, \xi, \mu, \varepsilon)$ solves the problem in an orthogonal space to $M$. Then, we are left with a finite dimensional problem, for which a solution may be found using the assumptions of the theorems. In the subcritical or critical case, the first step may be performed in $H^{1}$ (see e.g. $[4,31,32]$ ). However, this approach is not valid any more in the supercritical case, for $H^{1}$ does not inject into $L^{q}$ as $q>\frac{2 N}{N-2}$. In [9], a weighted Hölder spaces approach was used. In the present paper, we use weighted Sobolev spaces to reduce the problem to a finite dimensional one. 


\subsection{Boundary deformations}

Fix $a \in \partial \Omega$. We introduce a boundary deformation which strengthens the boundary near $a$. Without loss of generality, we may assume that $a=0$ and after rotation and translation of the coordinate system we may assume that the inward normal to $\partial \Omega$ at $a$ is the direction of the positive $x_{N}$-axis. Denote $x^{\prime}=\left(x_{1}, \ldots, x_{N-1}\right), B^{\prime}(\delta)=$ $\left\{x^{\prime} \in \mathbb{R}^{N-1}:\left|x^{\prime}\right|<\delta\right\}$, and $\Omega_{1}=\Omega \cap B(a, \delta)$, where $B(a, \delta)=\left\{x \in \mathbb{R}^{N}:|x-a|<\delta\right\}$.

Then, since $\partial \Omega$ is smooth, we can find a constant $\delta>0$ such that $\partial \Omega \cap B(a, \delta)$ can be represented by the graph of a smooth function $\rho_{a}: B^{\prime}(\delta) \rightarrow R$, where $\rho_{a}(0)=0, \nabla \rho_{a}(0)=0$, and

$$
\Omega \cap B(a, \delta)=\left\{\left(x^{\prime}, x_{N}\right) \in B(a, \delta): x_{N}>\rho_{a}\left(x^{\prime}\right)\right\} .
$$

Moreover, we may write

$$
\rho_{a}\left(x^{\prime}\right)=\frac{1}{2} \sum_{i=1}^{N-1} k_{i} x_{i}^{2}+O\left(|x|^{3}\right) .
$$

Here $k_{i}, i=1, \ldots, N-1$, are the principal curvatures at $a$. Furthermore, the average of the principal curvatures of $\partial \Omega$ at $a$ is the mean curvature $H(a)=\frac{1}{N-1} \sum_{i=1}^{N-1} k_{i}$. To avoid clumsy notations, we drop the index $a$ in $\rho$.

On $\partial \Omega \cap B(a, \delta)$, the normal derivative $n(x)$ writes as

$$
n(x)=\frac{1}{\sqrt{1+\left|\nabla^{\prime} \rho\right|^{2}}}\left(\nabla^{\prime} \rho,-1\right)
$$

and the tangential derivatives are given by

$$
\frac{\partial}{\partial \tau_{i, x}}=\frac{1}{\sqrt{1+\left|\partial \rho / \partial x_{i}\right|^{2}}}\left(0, \ldots, 1, \ldots, \frac{\partial \rho}{\partial x_{i}}\right), \quad i=1, \ldots, N-1 .
$$

When there is no confusion, we also drop the dependence of $\partial / \partial \tau_{i, x}$ on $x$.

\subsection{Expansion of $V$ and $W$}

In Appendix (Lemma A.1), we derive the following asymptotic expansion of $V$ : For $N \geqslant 4$, we have the expansion

$$
V=U_{\frac{1}{\Lambda \varepsilon}, a}-(\Lambda \varepsilon)^{\frac{4-N}{2}} \varphi_{0}\left(\frac{x-a}{\Lambda \varepsilon}\right)+O\left(\varepsilon^{\frac{6-N}{2}}|\ln \varepsilon|^{m}\right)
$$

where $\varphi_{0}$ solves some linear problem and $m=1$ for $N=4$ and $m=0$ for $N \geqslant 5$. This then implies that

$$
W=U_{\frac{1}{\Lambda}, \xi}(x)-\hat{\varphi}(x)
$$

where

$$
\hat{\varphi}(x)=\varepsilon \Lambda^{\frac{4-N}{2}} \varphi_{0}\left(\frac{x-\xi}{\Lambda}\right)+O\left(\varepsilon^{2}|\ln \varepsilon|^{m}\right) .
$$

Furthermore, we have the following upper bound

$$
|\hat{\varphi}(x)| \leqslant \frac{C \varepsilon|\ln , \varepsilon|^{n}}{(1+|x-\xi|)^{N-3}}, \quad x \in \Omega_{\varepsilon}
$$

where $n=1$ for $N=4,5$ and $n=0$ for $N \geqslant 6$, whence

$$
|W(x)| \leqslant C\left(U_{\frac{1}{\Lambda}, \xi}\right)^{1-\tau} \text { in } \Omega_{\varepsilon}
$$

where $\tau$ is a positive number which can be chosen to be zero as $N \geqslant 6$, and as small as desired as $N=4,5$. 


\section{The finite dimensional reduction}

\subsection{Inversion of the linearized problem}

We first consider the linearized problem at a function $W_{\Lambda, \xi, \mu, \varepsilon}$, and we invert it in an orthogonal space to $M$. From now on, we omit for sake of simplicity the indices in the writing of $W_{\Lambda, \xi, \mu, \varepsilon}$. Equipping $H^{1}\left(\Omega_{\varepsilon}\right)$ with the scalar product

$$
(u, v)_{\varepsilon}=\int_{\Omega_{\varepsilon}}\left(\nabla u \cdot \nabla v+\mu \varepsilon^{2} u v\right)
$$

orthogonality to the functions

$$
Y_{0}=\frac{\partial W}{\partial \Lambda}, \quad Y_{i}=\frac{\partial W}{\partial \tau_{i}}, \quad 1 \leqslant i \leqslant N-1,
$$

in that space is equivalent, setting

$$
Z_{0}=-\Delta \frac{\partial W}{\partial \Lambda}+\mu \varepsilon^{2} \frac{\partial W}{\partial \Lambda}, \quad Z_{i}=-\Delta \frac{\partial W}{\partial \tau_{i}}+\mu \varepsilon^{2} \frac{\partial W}{\partial \tau_{i}}, \quad 1 \leqslant i \leqslant N-1
$$

to the orthogonality in $L^{2}\left(\Omega_{\varepsilon}\right)$, equipped with the usual scalar product $\langle\cdot, \cdot\rangle$, to the functions $Z_{i}, 0 \leqslant i \leqslant N-1$. Then, we consider the following problem $: h$ being given, find a function $\phi$ which satisfies

$$
\begin{cases}-\Delta \phi+\mu \varepsilon^{2} \phi-\alpha_{N}\left(\frac{N+2}{N-2}+\varepsilon\right) W^{\frac{4}{N-2}+\varepsilon} \phi=h+\sum_{i} c_{i} Z_{i} & \text { in } \Omega_{\varepsilon}, \\ \frac{\partial \phi}{\partial n}=0 & \text { on } \partial \Omega_{\varepsilon}, \\ 0 \leqslant i \leqslant N-1, \quad\left\langle Z_{i}, \phi\right\rangle=0 & \end{cases}
$$

for some numbers $c_{i}$.

Existence and uniqueness of $\phi$ will follow from an inversion procedure in suitable functional spaces. For $N=3$, the weighted Hölder spaces in [9] or [34] work well. For $N \geqslant 4$, we use a weighted Sobolev approach which seems more suitable in treating the large dimensions case. (Special attention is needed for the case $N=4$.) Similar approach has been used in [39] in dealing with a slightly supercritical exponent problem.

Let $\mathcal{U}$ be an open set in $\mathbb{R}^{N}$ and $\xi \in \mathcal{U}$. For $1<t<+\infty$, a nonnegative integer $l$, and a real number $\beta$, we define a weighted Sobolev norm

$$
\|\phi\|_{W_{\beta}^{l, t}(\mathcal{U})}=\sum_{|\alpha|=0}^{l}\left\|\langle x-\xi\rangle^{\beta+|\alpha|} \partial^{\alpha} \phi\right\|_{L^{t}(\mathcal{U})}
$$

where $\langle x-\xi\rangle=\left(1+|x-\xi|^{2}\right)^{\frac{1}{2}}$. When $l=0$, we denote $W_{\beta}^{0, t}(\mathcal{U})$ as $L_{\beta}^{t}(\mathcal{U})$.

Let $f$ be a function in $\Omega_{\varepsilon}$. We define the following two weighted Sobolev norms

$$
\|f\|_{*}=\|f\|_{W_{\beta}^{2, t}\left(\Omega_{\varepsilon}\right)}
$$

and

$$
\|f\|_{* *}=\|f\|_{L_{\beta+2}^{t}\left(\Omega_{\varepsilon}\right)} .
$$

We choose $t$ and $\beta$ such that

$$
N<t<+\infty, \quad \frac{N-2}{2}+\frac{N(N-2)}{4 t}<\beta<\frac{N}{t^{\prime}}-2
$$


where $t^{\prime}$ is the conjugate exponent of $t$, i.e., $\frac{1}{t}+\frac{1}{t^{\prime}}=1$. (It is easily checked that such a choice of $t$ and $\beta$ is always possible.) Since $t>N$, by Sobolev embedding theorem, we have

$$
|\nabla \phi(x)|+|\phi(x)| \leqslant C\langle x-\xi\rangle^{-\beta}\|\phi\|_{*}, \quad \forall x \in \Omega_{\varepsilon} .
$$

We recall the following result:

Lemma 3.1 (Corollary 1 of [25]). The integral operator

$$
T u(x)=\int_{\mathbb{R}^{N}} \frac{u(y)}{|x-y|^{N-2}} \mathrm{~d} y
$$

is a bounded operator from $L_{\beta+2}^{t}\left(\mathbb{R}^{N}\right)$ to $L_{\beta}^{t}\left(\mathbb{R}^{N}\right)$, provided that $-\frac{N}{t}<\beta<\frac{N}{t^{\prime}}-2$.

We are also in need of the following lemma, whose proof is given in the Appendix:

Lemma 3.2. Let $f \in L_{\beta+2}^{t}\left(\Omega_{\varepsilon}\right)$ and $u$ satisfy

$$
-\Delta u+\mu \varepsilon^{2} u=f \quad \text { in } \Omega_{\varepsilon}, \quad \frac{\partial u}{\partial n}=0 \quad \text { on } \partial \Omega_{\varepsilon} .
$$

Then we have

$$
|u(x)| \leqslant C \int_{\Omega_{\varepsilon}} \frac{|f(y)|}{|x-y|^{N-2}} \mathrm{~d} y
$$

and

$$
\|u\|_{*} \leqslant C\|f\|_{* *}
$$

The main result of this subsection is:

Proposition 3.1. There exists $\varepsilon_{0}>0$ and a constant $C>0$, independent of $\varepsilon$ and $\xi, \Lambda$ satisfying (2.3), such that for all $0<\varepsilon<\varepsilon_{0}$ and all $h \in L_{\beta+2}^{t}\left(\Omega_{\varepsilon}\right)$, problem (3.3) has a unique solution $\phi \equiv L_{\varepsilon}(h)$. Besides,

$$
\left\|L_{\varepsilon}(h)\right\|_{*} \leqslant C\|h\|_{* *}, \quad\left|c_{i}\right| \leqslant C\|h\|_{* *} .
$$

Moreover, the map $L_{\varepsilon}(h)$ is $C^{1}$ with respect to $\Lambda, \xi$ and the $W_{\beta}^{2, t}\left(\Omega_{\varepsilon}\right)$-norm, and

$$
\left\|D_{(\Lambda, \xi)} L_{\varepsilon}(h)\right\|_{*} \leqslant C\|h\|_{* *} .
$$

Proof. The argument follows closely the ideas in [9] and [34]. We repeat it since we use a different norm. The proof relies on the following result:

Lemma 3.3. Assume that $\phi_{\varepsilon}$ solves (3.3) for $h=h_{\varepsilon}$. If $\left\|h_{\varepsilon}\right\|_{* *}$ goes to zero as $\varepsilon$ goes to zero, so does $\left\|\phi_{\varepsilon}\right\|_{*}$.

Proof of Lemma 3.3. Arguing by contradiction, we may assume that $\left\|\phi_{\varepsilon}\right\|_{*}=1$. Multiplying the first equation in (3.3) by $Y_{j}$ and integrating in $\Omega_{\varepsilon}$ we find

$$
\sum_{i} c_{i}\left\langle Z_{i}, Y_{j}\right\rangle=\left\langle-\Delta Y_{j}+\mu \varepsilon^{2} Y_{j}-\alpha_{N}\left(\frac{N+2}{N-2}+\varepsilon\right) W^{\frac{4}{N-2}+\varepsilon} Y_{j}, \phi_{\varepsilon}\right\rangle-\left\langle h_{\varepsilon}, Y_{j}\right\rangle .
$$

On one hand we check, in view of the definition of $Z_{i}, Y_{j}$

$$
\left\langle Z_{0}, Y_{0}\right\rangle=\left\|Y_{0}\right\|_{\varepsilon}^{2}=c_{0}+o(1), \quad\left\langle Z_{i}, Y_{i}\right\rangle=\left\|Y_{i}\right\|_{\varepsilon}^{2}=c_{1}+o(1), \quad 1 \leqslant i \leqslant N-1
$$


where $c_{0}, c_{1}$ are strictly positive constants, and

$$
\left\langle Z_{i}, Y_{j}\right\rangle=o(1), \quad i \neq j .
$$

On the other hand, in view of the definition of $Y_{j}$ and $W$, straightforward computations yield

$$
\left\langle-\Delta Y_{j}+\mu \varepsilon^{2} Y_{j}-\alpha_{N}\left(\frac{N+2}{N-2}+\varepsilon\right) W^{\frac{4}{N-2}+\varepsilon} Y_{j}, \phi_{\varepsilon}\right\rangle=o\left(\left\|\phi_{\varepsilon}\right\|_{*}\right)
$$

and

$$
\left\langle h_{\varepsilon}, Y_{j}\right\rangle=O\left(\left\|h_{\varepsilon}\right\|_{* *}\right) .
$$

Consequently, inverting the quasi diagonal linear system solved by the $c_{i}$ 's, we find

$$
c_{i}=O\left(\left\|h_{\varepsilon}\right\|_{* *}\right)+o\left(\left\|\phi_{\varepsilon}\right\|_{*}\right) .
$$

In particular, $c_{i}=o(1)$ as $\varepsilon$ goes to zero.

Since $\left\|\phi_{\varepsilon}\right\|_{*}=1$, elliptic theory shows that along some subsequence, $\tilde{\phi}_{\varepsilon}(x)=\phi_{\varepsilon}(x-\xi)$ converges uniformly in any compact subset of $\mathbb{R}_{+}^{N}$ to a nontrivial solution of

$$
-\Delta \tilde{\phi}=\alpha_{N} \frac{N+2}{N-2} U_{\tilde{\Lambda}, 0}^{\frac{4}{N-2}} \tilde{\phi}
$$

for some $\tilde{\Lambda}>0$. Moreover, $\tilde{\phi} \in L_{\beta}^{t}\left(\mathbb{R}^{N}\right)$. A bootstrap argument (see e.g. Proposition 2.2 of [39]) implies $|\tilde{\phi}(x)| \leqslant$ $C /|x|^{N-2}$. As a consequence, $\tilde{\phi}$ writes as

$$
\tilde{\phi}=\alpha_{0} \frac{\partial U_{\tilde{\Lambda}, 0}}{\partial \tilde{\Lambda}}+\sum_{i=1}^{N-1} \alpha_{i} \frac{\partial U_{\tilde{\Lambda}, 0}}{\partial a_{i}}
$$

(see [31]). On the other hand, equalities $\left\langle Z_{i}, \phi_{\varepsilon}\right\rangle=0$ provide us with the equalities

$$
\begin{aligned}
& \int_{\mathbb{R}_{+}^{N}}-\Delta \frac{\partial U_{\tilde{\Lambda}, 0}}{\partial \tilde{\Lambda}} \tilde{\phi}=\int_{\mathbb{R}_{+}^{N}} U_{\tilde{\Lambda}, 0}^{\frac{4}{N-2}} \frac{\partial U_{\tilde{\Lambda}, 0}}{\partial \tilde{\Lambda}} \tilde{\phi}=0, \\
& \int_{\mathbb{R}_{+}^{N}}-\Delta \frac{\partial U_{\tilde{\Lambda}, 0}}{\partial a_{i}} \tilde{\phi}=\int_{\mathbb{R}_{+}^{N}} U_{\tilde{\Lambda}, 0}^{\frac{4}{N-2}} \frac{\partial U_{\tilde{\Lambda}, 0}}{\partial a_{i}} \tilde{\phi}=0, \quad 1 \leqslant i \leqslant N-1 .
\end{aligned}
$$

As we have also

$$
\int_{\mathbb{R}_{+}^{N}}\left|\nabla \frac{\partial U_{\tilde{\Lambda}, 0}}{\partial \tilde{\Lambda}}\right|^{2}=c_{0}>0, \quad \int_{\mathbb{R}_{+}^{N}}\left|\nabla \frac{\partial U_{\tilde{\Lambda}, 0}}{\partial a_{i}}\right|^{2}=c_{1}>0, \quad 1 \leqslant i \leqslant N-1,
$$

and

$$
\int_{\mathbb{R}_{+}^{N}} \nabla \frac{\partial U_{\tilde{\Lambda}, 0}}{\partial \tilde{\Lambda}} \cdot \nabla \frac{\partial U_{\tilde{\Lambda}, 0}}{\partial a_{i}}=\int_{\mathbb{R}_{+}^{N}} \nabla \frac{\partial U_{\tilde{\Lambda}, 0}}{\partial a_{j}} \cdot \nabla \frac{\partial U_{\tilde{\Lambda}, 0}}{\partial a_{i}}=0, \quad i \neq j,
$$

the $\alpha_{j}$ 's solve a homogeneous quasi diagonal linear system, yielding $\alpha_{j}=0,0 \leqslant \alpha_{j} \leqslant N-1$, and $\tilde{\phi}=0$. So $\phi_{\varepsilon}(x-\xi) \rightarrow 0$ in $C_{\mathrm{loc}}^{1}\left(\Omega_{\varepsilon}\right)$. Now, since

$$
\left|\langle x-\xi\rangle^{\beta+2} W^{\frac{4}{N-2}+\varepsilon} \phi_{\varepsilon}\right|^{t} \leqslant C\left\|\phi_{\varepsilon}\right\|_{*}^{t}\langle x-\xi\rangle^{(2-(4+(N-2) \varepsilon)(1-\tau)) t} \in L^{1}\left(\mathbb{R}^{N}\right),
$$


(using (2.18)), by the Dominated Convergence Theorem we obtain

$$
\int_{\Omega_{\varepsilon}}\left|\langle x-\xi\rangle^{\beta+2} W^{\frac{4}{N-2}+\varepsilon} \phi_{\varepsilon}\right|^{t}=o(1) \quad \text { i.e. } \quad\left\|W^{\frac{4}{N-2}+\varepsilon} \phi_{\varepsilon}\right\|_{* *}=o(1) .
$$

On the other hand, from (2.6), (3.2) and the definition of $U$, we know that

$$
\langle x-\xi\rangle^{\beta+2}\left|Z_{i}\right| \leqslant C\langle x-\xi\rangle^{\beta-N} \in L^{t}\left(\mathbb{R}^{N}\right) .
$$

Applying Lemma 3.2 we obtain

$$
\left\|\phi_{\varepsilon}\right\|_{*} \leqslant C\left\|W^{\frac{4}{N-2}+\varepsilon} \phi_{\varepsilon}\right\|_{* *}+C\left\|h_{\varepsilon}\right\|_{* *}+C \sum_{i}\left|c_{i}\right|\left\|Z_{i}\right\|_{* *}=o(1)
$$

that is, a contradiction.

Proof of Proposition 3.1 completed. We set

$$
H=\left\{\phi \in H^{1}\left(\Omega_{\varepsilon}\right),\left\langle Z_{i}, \phi\right\rangle=0,0 \leqslant i \leqslant N-1\right\}
$$

equipped with the scalar product $(\cdot, \cdot)_{\varepsilon}$. Problem (3.3) is equivalent to finding $\phi \in H$ such that

$$
(\phi, \theta)_{\varepsilon}=\left\langle\alpha_{N}\left(\frac{N+2}{N-2}+\varepsilon\right) W^{\frac{4}{N-2}+\varepsilon} \phi+h, \theta\right\rangle, \quad \forall \theta \in H
$$

that is

$$
\phi=T_{\varepsilon}(\phi)+\tilde{h}
$$

$\tilde{h}$ depending linearly on $h$, and $T_{\varepsilon}$ being a compact operator in $H$. Fredholm's alternative ensures the existence of a unique solution, provided that the kernel of $\mathrm{Id}-T_{\varepsilon}$ is reduced to 0 . We notice that any $\phi_{\varepsilon} \in \operatorname{Ker}\left(\mathrm{Id}-T_{\varepsilon}\right)$ solves (3.3) with $h=0$. Thus, we deduce from Lemma 3.3 that $\left\|\phi_{\varepsilon}\right\|_{*}=o(1)$ as $\varepsilon$ goes to zero. As $\operatorname{Ker}\left(\operatorname{Id}-T_{\varepsilon}\right)$ is a vector space, $\operatorname{Ker}\left(\operatorname{Id}-T_{\varepsilon}\right)=\{0\}$. The inequalities (3.8) follow from Lemma 3.3 and (3.12). This completes the proof of the first part of Proposition 3.1.

The smoothness of $L_{\varepsilon}$ with respect to $\Lambda$ and $\xi$ is a consequence of the smoothness of $T_{\varepsilon}$ and $\tilde{h}$, which occur in the implicit definition (3.13) of $\phi \equiv L_{\varepsilon}(h)$, with respect to these variables. Inequalities (3.9) are obtained differentiating (3.3), writing the derivatives of $\phi$ with respect to $\Lambda$ and $\xi$ as a linear combination of the $Z_{i}$ ' and an orthogonal part, and estimating each term using the first part of the proposition - see $[9,19]$ for detailed computations.

\subsection{The reduction}

Let

$$
S_{\varepsilon}(u)=-\Delta u+\mu \varepsilon^{2} u-\alpha_{N} u_{+}^{\frac{N+2}{N-2}+\varepsilon}
$$

where $u_{+}=\max (0, u)$. Then (2.9) is equivalent to

$$
S_{\varepsilon}(u)=0 \quad \text { in } \partial \Omega_{\varepsilon}, \quad u_{+} \not \equiv 0, \quad \frac{\partial u}{\partial n}=0 \quad \text { on } \partial \Omega_{\varepsilon}
$$

for if $u$ satisfies (3.14), the Maximum Principle ensures that $u>0$ in $\Omega_{\varepsilon}$ and (2.9) is satisfied. Observe that

$$
S_{\varepsilon}(W+\phi)=-\Delta(W+\phi)+\mu \varepsilon^{2}(W+\phi)-\alpha_{N}(W+\phi)_{+}^{\frac{N+2}{N-2}+\varepsilon}
$$

may be written as

$$
S_{\varepsilon}(W+\phi)=-\Delta \phi+\mu \varepsilon^{2} \phi-\left(\frac{N+2}{N-2}+\varepsilon\right) \alpha_{N} W^{\frac{4}{N-2}+\varepsilon} \phi-R^{\varepsilon}-\alpha_{N} N_{\varepsilon}(\phi)
$$


with

$$
\begin{aligned}
& N_{\varepsilon}(\phi)=(W+\phi)_{+}^{\frac{N+2}{N-2}+\varepsilon}-W^{\frac{N+2}{N-2}+\varepsilon}-\left(\frac{N+2}{N-2}+\varepsilon\right) W^{\frac{4}{N-2}+\varepsilon} \phi \\
& R^{\varepsilon}=\Delta W-\mu \varepsilon^{2} W+\alpha_{N} W^{\frac{N+2}{N-2}+\varepsilon}=\alpha_{N}\left(W^{\frac{N+2}{N-2}+\varepsilon}-U_{\frac{1}{\Lambda}, \xi}^{\frac{N+2}{N-2}}\right) .
\end{aligned}
$$

We first have:

Lemma 3.4. There exists $C$, independent of $\xi, \Lambda$ satisfying (2.3), such that

$$
\left\|R^{\varepsilon}\right\|_{* *} \leqslant C \varepsilon, \quad\left\|D_{(\Lambda, \xi)} R^{\varepsilon}\right\|_{* *} \leqslant C \varepsilon .
$$

Proof. According to (2.15) and (2.18), $W=U+O\left(\varepsilon U^{\frac{N-3}{N-2}(1-\tau)}\right)$ uniformly in $\Omega_{\varepsilon}$ (where $\tau$ is a positive number which is either zero, or may be chosen as small as desired). Consequently, noticing that $U \geqslant C \varepsilon^{N-2}$ in $\Omega_{\varepsilon}, C$ independent of $\varepsilon$, easy computations yield

$$
R^{\varepsilon}=O\left(\varepsilon U^{\frac{N+2}{N-2}\left(1-\tau^{\prime}\right)}|\ln U|+\varepsilon U^{\frac{N+1}{N-2}\left(1-\tau^{\prime \prime}\right)}\right)
$$

uniformly in $\Omega_{\varepsilon}$ whence, using (3.4)

$$
\begin{aligned}
\left\|R^{\varepsilon}\right\|_{* *} & =\left\|\langle x-\xi\rangle^{\beta+2}\left(U^{\frac{N+2}{N-2}}-W^{\frac{N+2}{N-2}+\varepsilon}\right)\right\|_{L^{t}\left(\Omega_{\varepsilon}\right)} \\
& \leqslant C \varepsilon\left\|\langle x-\xi\rangle^{\beta+2}\left(U^{\frac{N+2}{N-2}\left(1-\tau^{\prime}\right)}|\ln U|+U^{\frac{N+1}{N-2}\left(1-\tau^{\prime \prime}\right)}\right)\right\|_{L^{t}\left(\Omega_{\varepsilon}\right)} \leqslant C \varepsilon .
\end{aligned}
$$

The first estimate of the lemma follows. The other ones are obtained in the same way, differentiating (3.17) and estimating each term as previously.

We consider now the following nonlinear problem: finding $\phi$ such that, for some numbers $c_{i}$

$$
\left\{\begin{array}{l}
-\Delta(W+\phi)+\mu \varepsilon^{2}(W+\phi)-\alpha_{N}(W+\phi)_{+}^{\frac{N+2}{N-2}+\varepsilon}=\sum_{i} c_{i} Z_{i} \quad \text { in } \Omega_{\varepsilon}, \\
\frac{\partial \phi}{\partial n}=0 \quad \text { on } \partial \Omega_{\varepsilon}, \\
0 \leqslant i \leqslant N-1, \quad\left\langle Z_{i}, \phi\right\rangle=0 .
\end{array}\right.
$$

The first equation in (3.19) writes as

$$
-\Delta \phi+\mu \varepsilon^{2} \phi-\left(\frac{N+2}{N-2}+\varepsilon\right) \alpha_{N} W^{\frac{4}{N-2}+\varepsilon} \phi=\alpha_{N} N_{\varepsilon}(\phi)+R^{\varepsilon}+\sum_{i} c_{i} Z_{i}
$$

for some numbers $c_{i}$. We now obtain some estimates concerning $N_{\varepsilon}$.

Lemma 3.5. Assume that $N \geqslant 4$ and (3.4) holds. There exist $\varepsilon_{1}>0$, independent of $\Lambda, \xi$, and $C$, independent of $\varepsilon, \Lambda, \xi$, such that for $|\varepsilon| \leqslant \varepsilon_{1}$, and $\|\phi\|_{*} \leqslant 1$

$$
\left\|N_{\varepsilon}(\phi)\right\|_{* *} \leqslant C\|\phi\|_{*}^{\min \left(2, \frac{N+2}{N-2}+\varepsilon\right)}
$$

and, for $\left\|\phi_{i}\right\|_{*} \leqslant 1$

$$
\left\|N_{\varepsilon}\left(\phi_{1}\right)-N_{\varepsilon}\left(\phi_{2}\right)\right\|_{* *} \leqslant C\left(\max \left(\left\|\phi_{1}\right\|_{*},\left\|\phi_{2}\right\|_{*}\right)\right)^{\min \left(1, \frac{4}{N-2}+\varepsilon\right)}\left\|\phi_{1}-\phi_{2}\right\|_{*} .
$$


Proof. The argument is similar to Lemma 3.1 and Proposition 3.5 of [39]. For the convenience of the reader, we include a proof here. We deduce from (3.16) that

$$
\begin{cases}\left|N_{\varepsilon}(\phi)\right| \leqslant C\left(W^{\frac{6-N}{N-2}+\varepsilon}|\phi|^{2}+|\phi|^{\frac{N+2}{N-2}+\varepsilon}\right) & \text { if } N \leqslant 6, \\ \left|N_{\varepsilon}(\phi)\right| \leqslant C|\phi|^{\frac{N+2}{N-2}+\varepsilon} & \text { if } N \geqslant 7 .\end{cases}
$$

Using (3.4) and (3.5) we have

$$
\begin{aligned}
\left\||\phi|^{\frac{N+2}{N-2}+\varepsilon}\right\|_{* *} & =\left(\int_{\Omega_{\varepsilon}}\left(\langle x-\xi\rangle^{\beta+2}|\phi|^{\frac{N+2}{N-2}+\varepsilon}\right)^{t}\right)^{\frac{1}{t}} \\
& \leqslant C\|\phi\|_{*}^{\frac{N+2}{N-2}+\varepsilon}\left(\int_{\Omega_{\varepsilon}}\langle x-\xi\rangle^{t\left(\beta+2-\left(\frac{N+2}{N-2}+\varepsilon\right) \beta\right)}\right)^{\frac{1}{t}} \leqslant C\|\phi\|_{*}^{\frac{N+2}{N-2}+\varepsilon} .
\end{aligned}
$$

For $N=4,5,6$, using also (2.18), and noticing that $W^{\varepsilon}$ is bounded since $W$ is bounded and satisfies (2.7)), we have

$$
\begin{aligned}
\left\|W^{\frac{6-N}{N-2}+\varepsilon}|\phi|^{2}\right\|_{* *} & =\left(\int_{\Omega_{\varepsilon}}\left(\langle x-\xi\rangle^{\beta+2} W^{\frac{6-N}{N-2}+\varepsilon}|\phi|^{2}\right)^{t}\right)^{\frac{1}{t}} \\
& \leqslant C\|\phi\|_{*}^{2}\left(\int_{\Omega_{\varepsilon}}\langle x-\xi\rangle^{(2-\beta+(N-6)(1-\tau)) t}\right)^{\frac{1}{t}} \leqslant C\|\phi\|_{*}^{2}
\end{aligned}
$$

whence (3.21). Concerning (3.22), we write

$$
N_{\varepsilon}\left(\phi_{1}\right)-N_{\varepsilon}\left(\phi_{2}\right)=\partial_{\eta} N_{\varepsilon}(\eta)\left(\phi_{1}-\phi_{2}\right)
$$

for some $\eta=x \phi_{1}+(1-x) \phi_{2}, x \in[0,1]$. From

$$
\partial_{\eta} N_{\varepsilon}(\eta)=\left(\frac{N+2}{N-2}+\varepsilon\right)\left((W+\eta)_{+}^{\frac{4}{N-2}+\varepsilon}-W^{\frac{4}{N-2}+\varepsilon}\right)
$$

we deduce

$$
\begin{cases}\left|\partial_{\eta} N_{\varepsilon}(\eta)\right| \leqslant C\left(W^{\frac{6-N}{N-2}+\varepsilon}|\eta|+|\eta|^{\frac{4}{N-2}+\varepsilon}\right) & \text { if } N \leqslant 6 \\ \left|\partial_{\eta} N_{\varepsilon}(\eta)\right| \leqslant C|\eta|^{\frac{4}{N-2}+\varepsilon} & \text { if } N \geqslant 7\end{cases}
$$

whence (3.22), using as previously (3.4) and (3.5).

We state now the following result:

Proposition 3.2. There exists $C$, independent of $\varepsilon$ and $\xi, \Lambda$ satisfying (2.3), such that for small $\varepsilon$ problem (3.19) has a unique solution $\phi=\phi(\Lambda, \xi, \mu, \varepsilon)$ with

$$
\|\phi\|_{*} \leqslant C \varepsilon .
$$

Moreover, $(\Lambda, \xi) \mapsto \phi(\Lambda, \xi, \mu, \varepsilon)$ is $C^{1}$ with respect to the $W_{\beta}^{2, t}\left(\Omega_{\varepsilon}\right)$-norm, and

$$
\left\|D_{(\Lambda, \xi)} \phi\right\|_{*} \leqslant C \varepsilon \text {. }
$$


Proof. Following [9], we consider the map $A_{\varepsilon}$ from $\mathcal{F}=\left\{\phi \in H^{1} \cap W_{\beta}^{2, t}\left(\Omega_{\varepsilon}\right):\|\phi\|_{*} \leqslant C_{0} \varepsilon\right\}$ to $H^{1} \cap W_{\beta}^{2, t}\left(\Omega_{\varepsilon}\right)$ defined as

$$
A_{\varepsilon}(\phi)=L_{\varepsilon}\left(\alpha_{N} N_{\varepsilon}(\phi)+R^{\varepsilon}\right)
$$

Here $C_{1}$ is a large number, to be determined later, and $L_{\varepsilon}$ is give by Proposition 3.1. We remark that finding a solution $\phi$ to problem (3.19) is equivalent to finding a fixed point of $A_{\varepsilon}$. One the one hand we have, for $\phi \in \mathcal{F}$ and $\varepsilon$ small enough

$$
\left\|A_{\varepsilon}(\phi)\right\|_{*} \leqslant\left\|L_{\varepsilon}\left(N_{\varepsilon}(\phi)\right)\right\|_{*}+\left\|L_{\varepsilon}\left(R^{\varepsilon}\right)\right\|_{*} \leqslant\left\|N_{\varepsilon}(\phi)\right\|_{* *}+C \varepsilon \leqslant 2 C \varepsilon
$$

with $C$ independent of $C_{0}$, implying that $A_{\varepsilon}$ sends $\mathcal{F}$ into itself, if we choose $C_{0}=2 C$. On the other hand $A_{\varepsilon}$ is a contraction. Indeed, for $\phi_{1}$ and $\phi_{2}$ in $\mathcal{F}$, we write

$$
\left\|A_{\varepsilon}\left(\phi_{1}\right)-A_{\varepsilon}\left(\phi_{2}\right)\right\|_{*} \leqslant C\left\|N_{\varepsilon}\left(\phi_{1}\right)-N_{\varepsilon}\left(\phi_{2}\right)\right\|_{* *} \leqslant C \varepsilon^{\min \left(1, \frac{4}{N-2}\right)}\left\|\phi_{1}-\phi_{2}\right\|_{*} \leqslant \frac{1}{2}\left\|\phi_{1}-\phi_{2}\right\|_{*}
$$

by Lemma (3.5). Contraction Mapping Theorem implies that $A_{\varepsilon}$ has a unique fixed point in $\mathcal{F}$, that is problem (3.19) has a unique solution $\phi$ such that $\|\phi\|_{*} \leqslant C_{0} \varepsilon$.

In order to prove that $(\Lambda, \xi) \mapsto \phi(\Lambda, \xi)$ is $C^{1}$, we remark that setting for $\eta \in \mathcal{F}$

$$
B(\Lambda, \xi, \eta) \equiv \eta-L_{\varepsilon}\left(\alpha_{N} N_{\varepsilon}(\eta)+R^{\varepsilon}\right)
$$

$\phi$ is defined as

$$
B(\Lambda, \xi, \phi)=0 .
$$

We have

$$
\partial_{\eta} B(\Lambda, \xi, \eta)[\theta]=\theta-\alpha_{N} L_{\varepsilon}\left(\theta\left(\partial_{\eta} N_{\varepsilon}\right)(\eta)\right) .
$$

Using Proposition 3.1, (3.5), (3.24) and (3.4) we obtain for $N \geqslant 7$

$$
\begin{aligned}
\left\|L_{\varepsilon}\left(\theta\left(\partial_{\eta} N_{\varepsilon}\right)(\eta)\right)\right\|_{*} & \leqslant C\left\|\theta\left(\partial_{\eta} N_{\varepsilon}\right)(\eta)\right\|_{* *} \leqslant C\left\|\langle x-\xi\rangle^{-\beta}\left(\partial_{\eta} N_{\varepsilon}\right)(\eta)\right\|_{* *}\|\theta\|_{*} \\
& \leqslant C\left\|\langle x-\xi\rangle^{2}|\eta|^{\frac{4}{N-2}+\varepsilon}\right\|_{L^{t}\left(\Omega_{\varepsilon}\right)}\|\theta\|_{*} \leqslant C\|\eta\|_{*}^{\frac{4}{N-2}+\varepsilon}\|\theta\|_{*} \\
& \leqslant C \varepsilon^{\frac{4}{N-2}}\|\theta\|_{*}
\end{aligned}
$$

and, proceeding in the same way, using also (2.18), we find as $N=4,5,6$

$$
\left\|L_{\varepsilon}\left(\theta\left(\partial_{\eta} N_{\varepsilon}\right)(\eta)\right)\right\|_{*} \leqslant C \varepsilon\|\theta\|_{*} .
$$

Therefore we can write, for any $N \geqslant 4$

$$
\left\|L_{\varepsilon}\left(\theta\left(\partial_{\eta} N_{\varepsilon}\right)(\eta)\right)\right\|_{*} \leqslant C \varepsilon^{\min \left(1, \frac{4}{N-2}\right)}\|\theta\|_{*} .
$$

Consequently, $\partial_{\eta} B(\Lambda, \xi, \phi)$ is invertible in $W_{\beta}^{2, t}\left(\Omega_{\varepsilon}\right)$ with uniformly bounded inverse. Then, the fact that $(\Lambda, \xi) \mapsto$ $\phi(\Lambda, \xi)$ is $C^{1}$ follows from the fact that $(\Lambda, \xi, \eta) \mapsto L_{\varepsilon}\left(N_{\varepsilon}(\eta)\right)$ is $C^{1}$ and the implicit functions theorem.

Finally, let us show how estimates (3.26) may be obtained. Derivating (3.27) with respect to $\Lambda$, we have

$$
\partial_{\Lambda} \phi=\left(\partial_{\eta} B(\Lambda, \xi, \phi)\right)^{-1}\left(\alpha_{N}\left(\partial_{\Lambda} L_{\varepsilon}\right)\left(N_{\varepsilon}(\phi)\right)+\alpha_{N} L_{\varepsilon}\left(\left(\partial_{\Lambda} N_{\varepsilon}\right)(\phi)\right)+\partial_{\Lambda}\left(L_{\varepsilon}\left(R^{\varepsilon}\right)\right)\right)
$$

whence, according to Proposition 3.1

$$
\begin{aligned}
\left\|\partial_{\Lambda} \phi\right\|_{*} & \leqslant C\left(\left\|\left(\partial_{\Lambda} L_{\varepsilon}\right)\left(N_{\varepsilon}(\phi)\right)\right\|_{*}+\left\|\left(L_{\varepsilon}\left(\partial_{\Lambda} N_{\varepsilon}\right)(\phi)\right)\right\|_{*}+\left\|\left(\partial_{\Lambda}\left(L_{\varepsilon}\left(R^{\varepsilon}\right)\right)\right)\right\|_{*}\right) \\
& \leqslant C\left(\left\|N_{\varepsilon}(\phi)\right\|_{* *}+\left\|\left(\partial_{\Lambda} N_{\varepsilon}\right)(\phi)\right\|_{* *}+\left\|\left(\partial_{\Lambda}\left(L_{\varepsilon}\left(R^{\varepsilon}\right)\right)\right)\right\|_{*}\right) .
\end{aligned}
$$


From (3.21) and (3.25) we know that

$$
\left\|N_{\varepsilon}(\phi)\right\|_{* *} \leqslant C \varepsilon^{\min \left(2, \frac{N+2}{N-2}\right)} .
$$

Concerning the next term, we notice that according to the definition (3.16) of $N_{\varepsilon}$ and the boundedness of $W^{\varepsilon}$

$$
\begin{aligned}
\left|\left(\partial_{\Lambda} N_{\varepsilon}\right)(\phi)\right| & \\
= & \left(\frac{N+2}{N-2}+\varepsilon\right)\left|(W+\phi)_{+}^{\frac{4}{N-2}+\varepsilon}-W^{\frac{4}{N-2}+\varepsilon}-\left(\frac{4}{N-2}+\varepsilon\right) W^{\frac{6-N}{N-2}+\varepsilon} \phi\right|\left|\partial_{\Lambda} W\right| \\
\leqslant & C\left[W^{\frac{4}{N-2}}|\phi| \text { if } N \geqslant 7 ; W^{\frac{4}{N-2}}|\phi|+W|\phi|^{\frac{4}{N-2}+\varepsilon} \text { if } N \leqslant 6\right] \\
\leqslant & C\left[\langle x-\xi\rangle^{-4(1-\tau)-\beta}\|\phi\|_{*} \text { if } N \geqslant 7 ;\right. \\
& \left.\langle x-\xi\rangle^{-4(1-\tau)-\beta}\|\phi\|_{*}+\langle x-\xi\rangle^{-(N-2)(1-\tau)-\frac{4}{N-2} \beta}\|\phi\|_{*}^{\frac{4}{N-2}+\varepsilon} \text { if } N \leqslant 6\right]
\end{aligned}
$$

where we used successively the fact that $W>0$ (see (2.7)) and $\left|\partial_{\Lambda} W\right| \leqslant C W$ (see (2.8)), inequality (3.5) and $W \leqslant C U^{1-\tau} \leqslant C\langle x-\xi\rangle^{-(N-2)(1-\tau)}$.

As (3.4) ensures that $\langle x-\xi\rangle^{-4(1-\tau)-\beta}$, and $\langle x-\xi\rangle^{-(N-2)(1-\tau)-\frac{4}{N-2} \beta}$ for $N \leqslant 6$, are in $L_{\beta+2}^{t}\left(\mathbb{R}^{N}\right)$ (provided that $\tau$ is chosen small enough), (3.25) yields

$$
\left\|\left(\partial_{\Lambda} N_{\varepsilon}\right)(\phi)\right\|_{* *} \leqslant C \varepsilon .
$$

From Proposition 3.1 we deduce the estimate for the last term

$$
\left\|\partial_{\Lambda}\left(L_{\varepsilon}\left(R^{\varepsilon}\right)\right)\right\|_{*} \leqslant C\left\|R^{\varepsilon}\right\|_{* *} \leqslant C \varepsilon
$$

and finally

$$
\left\|\partial_{\Lambda} \phi\right\|_{*} \leqslant C \varepsilon .
$$

This concludes the proof of Proposition 3.2. (The first derivatives of $\phi$ with respect to $\xi$ may be estimated in the same way, but this is not needed here.)

\subsection{Coming back to the original problem}

We introduce the following functional defined in $H^{1}\left(\Omega_{\varepsilon}\right) \cap W_{\beta}^{2, t}\left(\Omega_{\varepsilon}\right)$

$$
J_{\varepsilon}(u)=\frac{1}{2} \int_{\Omega_{\varepsilon}}\left(|\nabla u|^{2}+\mu \varepsilon^{2} u^{2}\right)-\frac{\alpha_{N}}{2 N /(N-2)+\varepsilon} \int_{\Omega_{\varepsilon}} u_{+}^{\frac{2 N}{N-2}+\varepsilon}
$$

whose nontrivial critical points are solutions to $\left(P_{\frac{N+2}{N-2}+\varepsilon, \mu}^{\prime}\right)$. Setting

$$
I_{\varepsilon}(\Lambda, a) \equiv J_{\varepsilon}\left(W_{\Lambda, a}+\phi_{\varepsilon, \Lambda, a}\right)
$$

we have:

Proposition 3.3. The function $u=W+\phi$ is a solution to problem $\left(P_{\frac{N+2}{N-2}+\varepsilon, \mu}^{\prime}\right)$ if and only if $(\Lambda, a)$ is a critical point of $I_{\varepsilon}$.

Proof. We notice that $u=W+\phi$ being a solution to $\left(P_{\frac{N+2}{N-2}+\varepsilon, \mu}^{\prime}\right)$ is equivalent to being a critical point of $J_{\varepsilon}$. It is also equivalent to the cancellation of the $c_{i}$ 's in (3.19) or, in view of (3.10), (3.11)

$$
J_{\varepsilon}^{\prime}(W+\phi)\left[Y_{i}\right]=0, \quad 0 \leqslant i \leqslant N-1 .
$$


On the other hand, we deduce from (3.29) that $I_{\varepsilon}^{\prime}(\Lambda, a)=0$ is equivalent to the cancellation of $J_{\varepsilon}^{\prime}(W+\phi)$ applied to the derivatives of $W+\phi$ with respect to $\Lambda$ and $\xi$. According to the definition (3.1) of the $Y_{i}$ 's, Lemma 3.4 and Proposition 3.2 we have

$$
\frac{\partial(W+\phi)}{\partial \Lambda}=Y_{0}+y_{0}, \quad \frac{\partial(W+\phi)}{\partial \xi_{j}}=Y_{j}+y_{j}, \quad 1 \leqslant j \leqslant N-1,
$$

with $\left\|y_{i}\right\|_{*}=o(1), 0 \leqslant i \leqslant N-1$. Writing

$$
y_{i}=y_{i}^{\prime}+\sum_{j=0}^{N-1} a_{i j} Y_{j}, \quad\left\langle y_{i}^{\prime}, Z_{j}\right\rangle=\left(y_{i}^{\prime}, Y_{j}\right)_{\varepsilon}=0, \quad 0 \leqslant i, j \leqslant N-1,
$$

and

$$
J_{\varepsilon}^{\prime}(W+\phi)\left[Y_{i}\right]=\alpha_{i}
$$

it turns out that $I_{\varepsilon}^{\prime}(\Lambda, a)=0$ is equivalent, since $J_{\varepsilon}^{\prime}(W+\phi)[\theta]=0$ for $\left\langle\theta, Z_{j}\right\rangle=\left(\theta, Y_{j}\right)_{\varepsilon}=0,0 \leqslant j \leqslant N-1$, to

$$
\left(\operatorname{Id}+\left[a_{i j}\right]\right)\left[\alpha_{i}\right]=0 .
$$

As $a_{i j}=O\left(\left\|y_{i}\right\|_{*}\right)=o(1)$, we see that $I_{\varepsilon}^{\prime}(\Lambda, a)=0$ means exactly that (3.30) is satisfied.

\section{Proofs of Theorems 1.1 and 1.2}

In view of Proposition 3.3 we have, for proving the theorem, to find critical points of $I_{\varepsilon}$. We establish first a $C^{1}$-expansion of $I_{\varepsilon}$.

\subsection{Expansion of $I_{\varepsilon}$}

Proposition 4.1. There exist $A, B, C$, strictly positive constants such that

$$
I_{\varepsilon}(\Lambda, a)=A-B \Lambda \varepsilon H(a)+\frac{(N-2)^{2}}{4} A \varepsilon \ln \Lambda+\varepsilon\left(C+\frac{(N-2)^{2}}{4 N} A\right)+\varepsilon \sigma_{\varepsilon}(\Lambda, a)
$$

with $\sigma_{\varepsilon}$ and $\partial_{\Lambda} \sigma_{\varepsilon}$ going to zero as $\varepsilon$ goes to zero, uniformly with respect to $\Lambda$ satisfying (2.3).

Proof. In Appendix, we shall prove

$$
J_{\varepsilon}(W)=A-B \Lambda \varepsilon H(a)+\frac{(N-2)^{2}}{4} A \varepsilon \ln \Lambda+\varepsilon\left(C+\frac{(N-2)^{2}}{4 N} A\right)+o(\varepsilon) .
$$

Then it remains to show that

$$
I_{\varepsilon}(\Lambda, a)-J_{\varepsilon}(W+\phi)=o(\varepsilon) .
$$

Actually, in view of (3.29), a Taylor expansion and the fact that $J_{\varepsilon}^{\prime}(W+\phi)[\phi]=0$ yield

$$
\begin{aligned}
I(\Lambda, a)-J_{\varepsilon}(W) & =J_{\varepsilon}(W+\phi)-J_{\varepsilon}(W)=-\int_{0}^{1} J_{\varepsilon}^{\prime \prime}(W+t \phi)[\phi, \phi] t \mathrm{~d} t \\
& =-\int_{0}^{1}\left(\int_{\Omega_{\varepsilon}}\left(|\nabla \phi|^{2}+\mu \varepsilon^{2} \phi^{2}-\alpha_{N}\left(\frac{N+2}{N-2}+\varepsilon\right)(W+t \phi)_{+}^{\frac{4}{N-2}+\varepsilon} \phi^{2}+R^{\varepsilon} \phi\right)\right) t \mathrm{~d} t
\end{aligned}
$$




$$
\begin{aligned}
= & -\int_{0}^{1}\left(\alpha_{N} \int_{\Omega_{\varepsilon}}\left(N_{\varepsilon}(\phi) \phi+\left(\frac{N+2}{N-2}+\varepsilon\right)\left[W^{\frac{4}{N-2}+\varepsilon}-(W+t \phi)_{+}^{\frac{4}{N-2}+\varepsilon}\right] \phi^{2}\right)\right) t \mathrm{~d} t \\
& -\frac{1}{2} \int_{\Omega_{\varepsilon}} R^{\varepsilon} \phi .
\end{aligned}
$$

The first term can be estimated as follows. Using (3.23), (3.5), (3.4) and Proposition 3.2, we have, for $N \geqslant 7$

$$
\left|\int_{\Omega_{\varepsilon}} N_{\varepsilon}(\phi) \phi\right| \leqslant C\|\phi\|_{*}^{\frac{2 N}{N-2}+\varepsilon} \int_{\Omega_{\varepsilon}}\langle x-\xi\rangle^{-\beta\left(\frac{2 N}{N-2}+\varepsilon\right)} \leqslant C \varepsilon^{\frac{2 N}{N-2}} .
$$

In the same way we obtain for $N=4,5,6$, in view of (3.23) and (2.18)

$$
\left|\int_{\Omega_{\varepsilon}} N_{\varepsilon}(\phi) \phi\right| \leqslant C \varepsilon^{\frac{2 N}{N-2}}+C\|\phi\|_{*}^{3} \int_{\Omega_{\varepsilon}}\langle x-\xi\rangle^{-3 \beta-(6-N)(1-\tau)} \leqslant C \varepsilon^{3}
$$

whence finally, for any $N \geqslant 4$

$$
\left|\int_{\Omega_{\varepsilon}} N_{\varepsilon}(\phi) \phi\right| \leqslant C \varepsilon^{\min \left(3, \frac{2 N}{N-2}\right)} .
$$

For the second term, the same arguments as previously yield

$$
\begin{aligned}
& \int_{\Omega_{\varepsilon}}\left|W^{\frac{4}{N-2}+\varepsilon}-(W+t \phi)_{+}^{\frac{4}{N-2}+\varepsilon}\right| \phi^{2} \leqslant C \int_{\Omega_{\varepsilon}}\left(W^{\frac{4}{N-2}+\varepsilon}|\phi|^{2}+|\phi|^{2+\frac{4}{N-2}+\varepsilon}\right) \\
& \leqslant C\left(\|\phi\|_{*}^{2} \int_{\Omega_{\varepsilon}}\langle x-\xi\rangle^{-2 \beta-4(1-\tau)}+\|\phi\|_{*}^{2+\frac{4}{N-2}+\varepsilon} \int_{\Omega_{\varepsilon}}\langle x-\xi\rangle^{-\beta\left(2+\frac{4}{N-2}+\varepsilon\right)}\right)
\end{aligned}
$$

whence, using again (3.4)

$$
\int_{\Omega_{\varepsilon}}\left|W^{\frac{4}{N-2}+\varepsilon}-(W+t \phi)_{+}^{\frac{4}{N-2}+\varepsilon}\right| \phi^{2} \leqslant C \varepsilon^{2} .
$$

Concerning the last term, we remark that according to (3.18)

$$
R^{\varepsilon} \leqslant C \varepsilon\langle x-\xi\rangle^{-(N+1)(1-\tau)}
$$

uniformly in $\Omega_{\varepsilon}$. Therefore

$$
\int_{\Omega_{\varepsilon}}\left|R^{\varepsilon} \phi\right| \leqslant C \varepsilon\|\phi\|_{*} \int_{\Omega_{\varepsilon}}\langle x-\xi\rangle^{-(N+1)-\beta}
$$

yielding, through Proposition 3.2

$$
\int_{\Omega_{\varepsilon}}\left|R^{\varepsilon} \phi\right| \leqslant C \varepsilon^{2} \text {. }
$$

The desired result follows from (4.3), (4.4) and (4.5). The same estimate holds for the first derivative with respect to $\Lambda$, obtained similarly with more delicate computations - see Proposition 3.4 of [19]. 


\subsection{Proofs of Theorem 1.1 and Theorem 1.2 completed}

We first prove Theorem 1.1 through a max-min argument. Since $\Omega$ is smooth and bounded, $\max _{P \in \partial \Omega} H(P)=$ $\gamma>0$. For $\delta<\gamma$, we define

$$
(\partial \Omega)_{\delta}=\{a \in \partial \Omega \text { s.t. } H(a)>\delta\},
$$

and

$$
\hat{I}_{\varepsilon}(\Lambda, a)=\frac{A-I_{\varepsilon}(\Lambda, a)}{B \varepsilon}+\frac{1}{B}\left(C+\frac{(N-2)^{2}}{4 N} A\right) .
$$

By Proposition 4.1, we have the following asymptotic expansion for $\hat{I}_{\epsilon}(\Lambda, a)$ :

$$
\hat{I}_{\varepsilon}(\Lambda, a)=\Lambda H(a)-\alpha \ln \Lambda-\tilde{\sigma}_{\varepsilon}(\Lambda, a)
$$

with

$$
\alpha=\frac{(N-2)^{2}}{4 B} A>0 \quad \text { and } \quad \tilde{\sigma}_{\varepsilon}(\Lambda, a)=o(1), \quad \partial_{\Lambda} \tilde{\sigma}_{\varepsilon}(\Lambda, a)=o(1) \quad \text { as } \varepsilon \rightarrow 0 .
$$

We set

$$
\Sigma_{0}=\left\{(\Lambda, a) \mid \frac{c_{1}}{2}<\Lambda<\frac{2}{c_{1}}, a \in(\partial \Omega)_{\gamma_{0}}\right\}
$$

where $c_{1}$ is a small number, to be chosen later, and $0<\gamma_{0}<\gamma$. We define also

$$
B=\left\{(\Lambda, a) \mid c_{1} \leqslant \Lambda \leqslant \frac{1}{c_{1}}, a \in(\partial \Omega)_{\gamma_{1}}\right\}, \quad B_{0}=\left\{c_{1}\right\} \times(\partial \Omega)_{\gamma_{1}} \cup\left\{\frac{1}{c_{1}}\right\} \times(\partial \Omega)_{\gamma_{1}}
$$

where $\gamma_{0}<\gamma_{1}<\gamma$. (Here we choose, for $\gamma_{1}$ close enough to $\gamma$, a contractible component of $(\partial \Omega)_{\gamma_{1}}$ so that $B$ is contractible.)

It is trivial to see that $B_{0} \subset B \subset \Sigma_{0}, B_{0}, B$ are closed and $B$ is connected. Let $\Gamma$ be the class of continuous functions $\varphi: B \rightarrow \Sigma_{0}$ with the property that $\varphi(y)=y$ for all $y \in B_{0}$. Define the max-min value $c$ as

$$
c=\max _{\varphi \in \Gamma} \min _{y \in B} \hat{I}_{\varepsilon}(\varphi(y)) \text {. }
$$

We now show that $c$ defines a critical value. To this end, we just have to verify the following two conditions

(H1) $\min _{y \in B_{0}} \hat{I}_{\varepsilon}(\varphi(y))>c, \forall \varphi \in \Gamma$;

(H2) For all $y \in \partial \Sigma_{0}$ such that $\hat{I}_{\epsilon}(y)=c$, there exists $\tau_{y}$ a tangent vector to $\partial \Sigma_{0}$ at $y$ such that

$$
\partial_{\tau_{y}} \hat{I}_{\epsilon}(y) \neq 0 \text {. }
$$

Suppose (H1) and (H2) hold. Then standard deformation argument ensures that the max-min value $c$ is a (topologically nontrivial ) critical value for $\hat{I}_{\varepsilon}(\Lambda, a)$ in $\Sigma_{0}$.

To check (H1) and (H2), we write $\varphi(y)=\left(\varphi_{1}(y), \varphi_{2}(y)\right)$ where $\varphi_{1}(y) \in\left[\frac{c_{1}}{2}, \frac{2}{c_{1}}\right]$ and $\varphi_{2}(y) \in(\partial \Omega)_{\gamma_{0}}$.

Since $\left.\varphi\right|_{B_{0}}=\mathrm{id}, B$ is contractible and $\varphi$ is continuous, necessarily there is some $y$ in $B$ such that $H\left(\varphi_{2}(y)\right)=\gamma$. Then, in view of (4.7)

$$
c \geqslant d_{0}:=\min \left\{\hat{I}_{\varepsilon}(\Lambda, a), H(a)=\gamma, \Lambda>0\right\}=\alpha-\alpha \ln \alpha+\alpha \ln \gamma+o(1) .
$$

Now, let $\left(\Lambda_{0}, a_{0}\right) \in B$ be such that $H\left(a_{0}\right)=\gamma, \Lambda_{0}=\frac{\alpha}{\gamma}\left(c_{1}\right.$ being chosen small enough so that $\left.\Lambda_{0} \in\left[c_{1}, \frac{1}{c_{1}}\right]\right)$. We note that $\hat{I}_{\varepsilon}\left(\Lambda_{0}, a_{0}\right)=d_{0}+o(1)$. For any $\varphi \in \Gamma, \varphi_{1}$ is a continuous function from B to $\left[\frac{c_{1}}{2}, \frac{2}{c_{1}}\right]$ such that $\left[c_{1}, \frac{1}{c_{1}}\right] \subset \varphi_{1}(B)$. Thus, there exists $y_{0} \in B$ such that $\varphi_{1}\left(y_{0}\right)=\Lambda_{0}$, whence 


$$
\min _{y \in B} \hat{I}_{\varepsilon}(\varphi(y)) \leqslant \hat{I}_{\varepsilon}\left(\Lambda_{0}, \varphi_{2}\left(y_{0}\right)\right) \leqslant \frac{\alpha}{\gamma} H\left(\varphi_{2}\left(y_{0}\right)\right)-\alpha \ln \alpha+\alpha \ln \gamma+o(1) \leqslant d_{0}=o(1) .
$$

As a consequence

$$
c=d_{0}+o(1)=\alpha-\alpha \ln \alpha+\alpha \ln \gamma+o(1) .
$$

For $y \in B_{0}$, we have $\varphi_{1}(y)=c_{1}$ or $\varphi_{1}(y)=\frac{1}{c_{1}}$. In the first case, we have $\hat{I}_{\varepsilon}(y)=c_{1} H\left(\varphi_{2}(y)\right)-\alpha \ln c_{1}+o(1)>$ $\alpha \ln \frac{1}{c_{1}}+o(1)>2 d_{0}>c$, provided $c_{1}$ is small enough. In the latter case, we have $\hat{I}_{\varepsilon}(y)=\frac{1}{c_{1}} H\left(\varphi_{2}(y)\right)+\alpha \ln c_{1}+$ $o(1)>\frac{\gamma_{1}}{c_{1}}+\alpha \ln c_{1}+o(1)>2 d_{0}>c$, provided again $c_{1}$ is small enough. So (H1) is verified.

To check $(H 2)$, we observe that $\partial\left(\Sigma_{0}\right)=\left(\left\{\frac{c_{1}}{2}\right\} \times(\partial \Omega)_{\gamma_{0}}\right) \cup\left(\left\{\frac{2}{c_{1}}\right\} \times(\partial \Omega)_{\gamma_{0}}\right) \cup\left(\left[c_{1}, \frac{1}{c_{1}}\right] \times\left(\partial(\partial \Omega)_{\gamma_{0}}\right)\right)$. Let $y=\left(y_{1}, y_{2}\right) \in \partial \Sigma_{0}$ be such that $\hat{I}_{\varepsilon}(y)=c$.

On $\left(\left\{\frac{c_{1}}{2}\right\} \times(\partial \Omega)_{\gamma_{0}}\right) \cup\left(\left\{\frac{2}{c_{1}}\right\} \times(\partial \Omega)_{\gamma_{0}}\right)$, previous arguments show that $\hat{I}_{\varepsilon}(y)>c$ as $c_{1}$ is chosen sufficiently small. On $\left(\left[c_{1}, \frac{1}{c_{1}}\right] \times\left(\partial\left((\partial \Omega)_{\gamma_{0}}\right)\right)\right.$, taking $\tau_{y}=\frac{\partial}{\partial \Lambda}$, we obtain

$$
\partial_{\tau_{y}} \hat{I}_{\epsilon}(y)=H\left(y_{2}\right)-\frac{\alpha}{\Lambda}+o(1) \neq 0
$$

since $\partial_{\tau_{y}} \hat{I}_{\epsilon}(y)=0$ would yield $\Lambda H\left(y_{2}\right)=\alpha+o(1)$, and

$$
\hat{I}_{\varepsilon}(y)=\alpha-\alpha \ln \alpha+\alpha \ln H\left(\varphi_{2}(y)\right)+o(1)=\alpha-\alpha \ln \alpha+\alpha \ln \gamma_{0}+o(1) .
$$

Then, (4.10) shows that $\hat{I}_{\varepsilon}(y)<c$, a contradiction to the assumption. So (H2) is also verified.

In conclusion, we proved that for $\varepsilon$ small enough, $c$ is a critical value, i.e. a critical point $\left(\Lambda_{\varepsilon}, a_{\varepsilon}\right) \in \Sigma_{0}$ of $\hat{I}_{\varepsilon}$ exists. Let $u_{\varepsilon}=W_{\Lambda_{\varepsilon}, \xi_{\varepsilon}, \mu, \varepsilon}+\phi_{\Lambda_{\varepsilon}, \xi_{\varepsilon}, \mu, \varepsilon} . u_{\varepsilon}$ is a nontrivial solution to the problem

$$
-\Delta u+\mu \varepsilon^{2} u=u_{+}^{\frac{N+2}{N-2}+\varepsilon} \quad \text { in } \Omega_{\varepsilon} ; \quad \frac{\partial u}{\partial n}=0 \quad \text { on } \partial \Omega_{\varepsilon} .
$$

Then, the strong maximum principle shows that $u_{\varepsilon}>0$ in $\Omega_{\varepsilon}$. The fact that $u_{\varepsilon}$ blows up, as $\varepsilon$ goes to zero, at a point $a$ such that $H(a)=\max _{P \in \partial \Omega} H(P)$, follows from the construction of $u_{\varepsilon}$. This concludes the proof of Theorem 1.1.

In the case of $\varepsilon<0$, we have

$$
\hat{I}_{\varepsilon}(\Lambda, a)=\Lambda H(a)+\alpha \ln (\Lambda)-\tilde{\sigma}_{\varepsilon}(\Lambda, a) .
$$

We assume that $\Omega$ is nonconvex. Similarly as before, we define

$$
(\partial \Omega)_{\delta}=\{a \in \partial \Omega \mid H(a)<-\delta\}
$$

where $0<\delta<\gamma=-\min _{a \in \partial \Omega} H(a)>0$, and

$$
\begin{array}{ll}
\Sigma_{0}=\left\{(\Lambda, a) \mid \frac{c_{1}}{2} \leqslant \Lambda \leqslant \frac{2}{c_{1}}, a \in(\partial \Omega)_{\gamma_{0}}\right\}, & \\
B=\left\{(\Lambda, a) \mid c_{1} \leqslant \Lambda \leqslant \frac{1}{c_{1}}, a \in(\partial \Omega)_{\gamma_{1}}\right\}, & B_{0}=\left\{c_{1}\right\} \times(\partial \Omega)_{\gamma} \cup\left\{\frac{1}{c_{1}}\right\} \times(\partial \Omega)_{\gamma_{1}}
\end{array}
$$

with $\gamma_{0}<\gamma_{1}<\gamma$.

Let $\Gamma$ be the class of continuous functions $\varphi: B \rightarrow \Sigma_{0}$ with the property that $\varphi(y)=y$ for all $y \in B_{0}$. We define the min-max value $c$ as

$$
c=\min _{\varphi \in \Gamma} \max _{y \in B} \hat{I}_{\varepsilon}(\varphi(y)) .
$$

Arguing as previously, we find that $c$ is a critical point of $\hat{I}_{\mathcal{E}}$. This proves Theorem 1.2. 


\section{Appendix}

\section{A.1. Error estimates}

We recall that, according to the definition of $V_{\Lambda, a, \mu, \varepsilon}$ in Section 2

$$
V_{\Lambda, a, \mu, \varepsilon}(x)=U_{\frac{1}{\Lambda \varepsilon}, a}(x)-\varphi_{\Lambda, a, \mu, \varepsilon}
$$

with $\varphi_{\Lambda, a, \mu, \varepsilon}$ satisfying

$$
\begin{cases}-\Delta \varphi_{\Lambda, a, \mu, \varepsilon}+\mu \varphi_{\Lambda, a, \mu, \varepsilon}=\mu U_{\frac{1}{\Lambda \varepsilon}, a} & \text { in } \Omega, \\ \frac{\partial \varphi_{\Lambda, a, \mu, \varepsilon}}{\partial n}=\frac{\partial U_{\frac{1}{\Lambda \varepsilon}, a}}{\partial n} & \text { on } \partial \Omega .\end{cases}
$$

This subsection is devoted to an expansion of $\varphi_{\Lambda, a, \mu, \varepsilon}$.

We recall that, through space translation and rotation, we assume that $a=0$ and $\Omega$ is given, in a neighborhood of $a$, by (2.10) and (2.11). We introduce an auxiliary function $\varphi_{0}$ : let $\varphi_{0}$ be such that

$$
\begin{cases}\Delta \varphi_{0}=0 & \text { in } \mathbb{R}_{+}^{N}=\left\{\left(x^{\prime}, x_{N}\right), x_{N}>0\right\}, \\ \frac{\partial \varphi_{0}}{\partial x_{N}}=\frac{N-2}{2} \frac{\sum_{i=1}^{N-1} k_{i} x_{i}^{2}}{\left(1+|x|^{2}\right)^{\frac{N}{2}}} & \text { on } \partial \mathbb{R}_{+}^{N}, \\ \varphi_{0}(x) \rightarrow 0 & \text { as }|x| \rightarrow+\infty .\end{cases}
$$

Using Green's representation, $\varphi_{0}$ writes as

$$
\varphi_{0}(x)=\frac{1}{\omega_{N-1}} \sum_{i=1}^{N-1} k_{i} \int_{\mathbb{R}^{N-1}} \frac{y_{i}^{2}}{\left(1+\left|y^{\prime}\right|^{2}\right)^{\frac{N}{2}}} \frac{1}{\left|x-y^{\prime}\right|^{N-2}} \mathrm{~d} y^{\prime}
$$

where $\omega_{N-1}$ denotes the measure of the unit sphere in $\mathbb{R}^{N}$. From (A.4) we deduce that

$$
\left|\varphi_{0}(x)\right| \leqslant \frac{C}{(1+|x|)^{N-3}}
$$

and

$$
\left|\nabla \varphi_{0}(x)\right| \leqslant \frac{C}{(1+|x|)^{N-2}}, \quad\left|D^{2} \varphi_{0}(x)\right| \leqslant \frac{C}{(1+|x|)^{N-1}} .
$$

Definition. From now on, we consider $\varphi_{0}$ as a smooth continuation in $\mathbb{R}^{N}$ of the previous function defined in $\mathbb{R}_{+}^{N}$, such that (A.5), (A.6) hold in whole $\mathbb{R}^{N}$.

We state:

Lemma A.1. For $N \geqslant 4$, we have the expansion

$$
\varphi_{\Lambda, a, \mu, \varepsilon}(x)=(\Lambda \varepsilon)^{\frac{4-N}{2}} \varphi_{0}\left(\frac{x-a}{\Lambda \varepsilon}\right)+O\left(\varepsilon^{\frac{6-N}{2}}|\ln \varepsilon|^{m}\right)
$$

with $m=1$ for $N=4$ and $m=0$ for $N \geqslant 5$. Moreover,

$$
\left|\varphi_{\Lambda, a, \mu, \varepsilon}(x)\right| \leqslant C \frac{\varepsilon^{\frac{4-N}{2}}|\ln \varepsilon|^{n}}{(1+|(x-a) /(\Lambda \varepsilon)|)^{N-3}} \quad \text { and } \quad\left|\varphi_{\Lambda, a, \mu, \varepsilon}(x)\right| \leqslant C\left(U_{\frac{1}{\Lambda \varepsilon}, a}(x)\right)^{1-\tau}
$$

with $n=1$ and $\tau>0$ is any small fixed number for $N=4,5, n=0$ and $\tau=0$ for $N \geqslant 6$. 
Proof. We first remark that the second inequality in (A.8) is a straightforward consequence of the first one. Next, we decompose

$$
\varphi=\varphi^{1}+\varphi^{2}
$$

where $\varphi^{1}$ satisfies

$$
\begin{cases}-\Delta \varphi_{\Lambda, a, \mu, \varepsilon}^{1}+\mu \varphi_{\Lambda, a, \mu, \varepsilon}^{1}=0 & \text { in } \Omega \\ \frac{\partial \varphi_{\Lambda, a, \mu, \varepsilon}^{1}}{\partial n}=\frac{\partial U_{\frac{1}{\Lambda \varepsilon}, a}}{\partial n} & \text { on } \partial \Omega\end{cases}
$$

and $\varphi^{2}$ satisfies

$$
\begin{cases}-\Delta \varphi_{\Lambda, a, \mu, \varepsilon}^{2}+\mu \varphi_{\Lambda, a, \mu, \varepsilon}^{2}=\mu U_{\frac{1}{\Lambda \varepsilon}, a} & \text { in } \Omega \\ \frac{\partial \varphi_{\Lambda, a, \mu, \varepsilon}^{2}}{\partial n}=0 & \text { on } \partial \Omega .\end{cases}
$$

Let us estimate $\varphi^{2}$ first. Let

$$
\hat{\varphi}^{j}(x)=\varepsilon^{\frac{N-2}{2}} \varphi^{j}(\varepsilon x) .
$$

Then $\hat{\varphi}^{2}$ satisfies

$$
\begin{cases}-\Delta \hat{\varphi}_{\Lambda, a, \mu, \varepsilon}^{2}+\mu \varepsilon^{2} \hat{\varphi}_{\Lambda, a, \mu, \varepsilon}^{2}=\mu \varepsilon^{2} U_{\frac{1}{\Lambda}, \xi} & \text { in } \Omega_{\varepsilon}, \\ \frac{\partial \hat{\varphi}_{\Lambda, a, \mu, \varepsilon}^{2}}{\partial n}=0 & \text { on } \partial \Omega_{\varepsilon} .\end{cases}
$$

Inequality (3.6) of Lemma 3.2 provides us with

$$
\left|\hat{\varphi}^{2}(x)\right| \leqslant C \varepsilon^{2} \int_{\Omega_{\varepsilon}} \frac{U_{\frac{1}{A}}, \xi}{|x-y|^{N-2}} \mathrm{~d} y \leqslant C \varepsilon^{2} \int_{\Omega_{\varepsilon}} \frac{d y}{(1+|y-\xi|)^{N-2}|x-y|^{N-2}}
$$

whence

$$
\left|\hat{\varphi}^{2}(x)\right| \leqslant C \frac{\varepsilon^{2}|\ln \varepsilon|^{m}}{(1+|x-\xi|)^{N-4}}
$$

with $m=1$ for $N=4$ and $m=0$ for $N \geqslant 5$. (For $N \geqslant 5$, see Lemma 2.3 of [21].) Consequently

$$
\varphi^{2}(x)=O\left(\varepsilon^{\frac{6-N}{2}}|\ln \varepsilon|^{m}\right) \quad \text { and } \quad\left|\varphi^{2}(x)\right| \leqslant C \frac{\varepsilon^{\frac{4-N}{2}}|\ln \varepsilon|^{m}}{(1+|(x-a) /(\Lambda \varepsilon)|)^{N-3}} .
$$

This finishes the estimate for $\varphi^{2}$. Next we estimate $\varphi^{1}$. To this end, we write

$$
\varphi_{\Lambda, a, \mu, \varepsilon}^{1}=(\Lambda \varepsilon)^{\frac{4-N}{2}} \varphi_{0}\left(\frac{x-a}{\Lambda \varepsilon}\right)+\varphi_{\Lambda, a, \mu, \varepsilon}^{3}(x)+\varphi_{\Lambda, a, \mu, \varepsilon}^{4}(x)
$$

where $\varphi_{\Lambda, a, \mu, \varepsilon}^{3}$ satisfies

$$
\begin{cases}-\Delta \varphi_{\Lambda, a, \mu, \varepsilon}^{3}+\mu \varphi_{\Lambda, a, \mu, \varepsilon}^{3}=0 & \text { in } \Omega \\ \frac{\partial \varphi_{\Lambda, a, \mu, \varepsilon}^{3}}{\partial n}=\frac{\partial U_{\frac{1}{\Lambda \varepsilon}, a}}{\partial n}-\frac{\partial}{\partial n}\left((\Lambda \varepsilon)^{\frac{4-N}{2}} \varphi_{0}\left(\frac{x-a}{\Lambda \varepsilon}\right)\right) & \text { on } \partial \Omega\end{cases}
$$


and $\varphi_{\Lambda, a, \mu, \varepsilon}^{4}$ satisfies

$$
\begin{cases}-\Delta \varphi_{\Lambda, a, \mu, \varepsilon}^{4}+\mu \varphi_{\Lambda, a, \mu, \varepsilon}^{4}=(\Delta-\mu)\left((\Lambda \varepsilon)^{\frac{4-N}{2}} \varphi_{0}\left(\frac{x-a}{\Lambda \varepsilon}\right)\right) & \text { in } \Omega, \\ \frac{\partial \varphi_{\Lambda, a, \mu, \varepsilon}^{4}=0}{\partial n}=0 & \text { on } \partial \Omega .\end{cases}
$$

The estimate for $\varphi^{4}$ is similar to that of $\varphi^{2}$. Namely, in view of (A.3) and (A.4), inequality (3.6) of Lemma 3.2 gives

$$
\begin{aligned}
\left|\hat{\varphi}^{4}(x)\right| & \leqslant C \varepsilon^{3}\left(\frac{1}{\varepsilon^{2}} \int_{\Omega_{\varepsilon} \mid \mathbb{R}_{+}^{N}} \frac{\mathrm{d} y}{(1+|y-\xi|)^{N-1}|x-y|^{N-2}}+\int_{\Omega_{\varepsilon}} \frac{\mathrm{d} y}{(1+|y-\xi|)^{N-3}|x-y|^{N-2}} \mathrm{~d} y\right) \\
& \leqslant C \varepsilon^{3}\left(\frac{1}{\varepsilon(1+|x-\xi|)^{N-3}}+\frac{|\ln \varepsilon|^{p}}{(1+|x-\xi|)^{N-5}}\right)
\end{aligned}
$$

with $p=1$ for $N=5$ and $p=0$ for $N \neq 5$, whence

$$
\varphi^{4}(x)=O\left(\varepsilon^{\frac{6-N}{2}}\right) \quad \text { and } \quad\left|\varphi^{4}(x)\right| \leqslant C \frac{\varepsilon^{\frac{4-N}{2}}|\ln \varepsilon|^{p}}{(1+|(x-a) /(\Lambda \varepsilon)|)^{N-3}} .
$$

It only remains to estimate $\varphi^{3}$. For $x \in \partial \Omega \cap B(a, \delta)$, we consider the following change of variable (still assuming $a=0$ )

$$
\Lambda \varepsilon y^{\prime}=x^{\prime}, \quad \Lambda \varepsilon y_{N}=x_{N}-\rho\left(x^{\prime}\right) .
$$

According to the definition of $U$ and (2.12), we have

$$
\begin{aligned}
\frac{\partial U_{\frac{1}{\Lambda \varepsilon}}, a}{\partial n}(x) & =-(N-2)(\Lambda \varepsilon)^{\frac{N-2}{2}} \frac{\langle x-a, n\rangle}{\left((\Lambda \varepsilon)^{2}+|x-a|^{2}\right)^{\frac{N}{2}}} \\
& =-\frac{N-2}{2} \frac{(\Lambda \varepsilon)^{\frac{N-2}{2}}}{\left((\Lambda \varepsilon)^{2}+|x-a|^{2}\right)^{\frac{N}{2}}}\left(\sum_{i=1}^{N-1} k_{i} x_{i}^{2}+O\left(\left|x^{\prime}\right|^{3}\right)\right) \\
& =-\frac{N-2}{2} \frac{(\Lambda \varepsilon)^{\frac{2-N}{2}}}{\left(1+\left|y^{\prime}\right|^{2}\right)^{\frac{N}{2}}}\left(\sum_{i=1}^{N-1} k_{i} y_{i}^{2}+O\left(\varepsilon\left|y^{\prime}\right|^{3}\right)\right)
\end{aligned}
$$

and, using (A.3) and (A.6)

$$
\begin{aligned}
\frac{\partial}{\partial n}\left((\Lambda \varepsilon)^{\frac{4-N}{2}} \varphi_{0}\left(\frac{x-a}{\Lambda \varepsilon}\right)\right) & =(\Lambda \varepsilon)^{\frac{2-N}{2}}\left(\nabla^{\prime} \varphi_{0}\left(\frac{x-a}{\Lambda \varepsilon}\right) \cdot \nabla^{\prime} \rho(x)-\frac{\partial \varphi_{0}}{\partial x_{N}}\left(\frac{x-a}{\Lambda \varepsilon}\right)\right) \\
& =-\frac{N-2}{2} \frac{(\Lambda \varepsilon)^{\frac{2-N}{2}}}{\left(1+\left|y^{\prime}\right|^{2}\right)^{\frac{N}{2}}} \sum_{i=1}^{N-1} k_{i} y_{i}^{2}+O\left(\frac{\varepsilon^{\frac{4-N}{2}}\left|y^{\prime}\right|}{\left(1+\left|y^{\prime}\right|\right)^{N-2}}\right)
\end{aligned}
$$

Therefore

$$
\frac{\partial \hat{\varphi}^{3}}{\partial n_{x}}(x)=\varepsilon^{\frac{N}{2}} \frac{\partial \varphi^{3}}{\partial n_{\varepsilon x}}(\varepsilon x)=O\left(\frac{\varepsilon^{2}\left|x^{\prime}\right|}{\left(1+\left|x^{\prime}\right|\right)^{N-2}}\right) \text { for } x \in \partial \Omega_{\varepsilon} \cap B\left(a, \frac{\delta}{\varepsilon}\right) .
$$

On the other hand we have clearly, from (A.6) and the definition of $U$

$$
\frac{\partial \hat{\varphi}^{3}}{\partial n}(x)=O\left(\varepsilon^{N-1}\right) \quad \text { for } x \in \partial \Omega_{\varepsilon} \cap B^{c}\left(a, \frac{\delta}{\varepsilon}\right) .
$$


Then, standard elliptic theory shows that $\hat{\varphi}^{3}=O\left(\varepsilon^{2}\right)$ uniformly in $\Omega_{\varepsilon}$, whence $\varphi^{3}(x)=O\left(\varepsilon^{\frac{6-N}{2}}\right)$ uniformly in $\Omega$. Moreover, (A.9) and (A.10) lead, through Green's representation, to the estimate

$$
\left|\hat{\varphi}^{3}(x)\right| \leqslant C \frac{\varepsilon^{2}}{(1+|x-\xi|)^{N-4}}
$$

whence

$$
\left|\varphi^{3}(x)\right| \leqslant C \frac{\varepsilon^{\frac{4-N}{2}}}{(1+|(x-a) /(\Lambda \varepsilon)|)^{N-3}} .
$$

This concludes the proof of Lemma A.1.

\section{A.2. Integral estimates}

Omitting, for sake of simplicity, the indices $\Lambda, a, \mu, \varepsilon$, we state:

Proposition A.1. $N \geqslant 4$. Assuming that $\Lambda$ satisfies (2.3), we have the uniform expansions as $\varepsilon$ goes to zero

$$
\begin{aligned}
& J_{\varepsilon}(W)=A-B \Lambda|\varepsilon| H(a)+\frac{(N-2)^{2} A}{4} \varepsilon \ln \Lambda+\left(C+\frac{(N-2)^{2} A}{4 N}\right) \varepsilon+O\left(\varepsilon^{2-\tau}\right), \\
& \frac{\partial J_{\varepsilon}}{\partial \Lambda}(W)=\frac{(N-2)^{2} A \varepsilon}{4 \Lambda}-B H(a)|\varepsilon|+O\left(\varepsilon^{2-\tau}\right)
\end{aligned}
$$

with

$$
A=(N-2) \int_{\mathbb{R}_{+}^{N}} U_{1,0}^{\frac{2 N}{N-2}} \quad C=-\frac{(N-2)^{2}}{2} \int_{\mathbb{R}_{+}^{N}} U_{1,0}^{\frac{2 N}{N-2}} \ln U_{1,0}>0
$$

and

$$
B=\frac{(N-2)^{2}}{N-3} \int_{\partial \mathbb{R}_{+}^{N}} U_{1,0}^{\frac{2 N}{N-2}}|y|^{2}
$$

Proof. For sake of simplicity, we assume that $\varepsilon>0$ (the computations are equivalent as $\varepsilon<0$ ). In view of (A.2) and (2.15), we write

$$
\int_{\Omega_{\varepsilon}}\left(|\nabla W|^{2}+\mu \varepsilon^{2} W^{2}\right)=\int_{\Omega_{\varepsilon}}\left(-\Delta W+\mu \varepsilon^{2} W\right) W=\int_{\Omega_{\varepsilon}} \alpha_{N} U^{\frac{N+2}{N-2}} W=\alpha_{N} \int_{\Omega_{\varepsilon}} U^{\frac{2 N}{N-2}}-\alpha_{N} \int_{\Omega_{\varepsilon}} U^{\frac{N+2}{N-2}} \hat{\varphi} .
$$

with $U=U_{\frac{1}{\Lambda}, \xi}$. On the other hand

$$
\begin{aligned}
\int_{\Omega_{\varepsilon}} W^{\frac{2 N}{N-2}+\varepsilon} & =\int_{\Omega_{\varepsilon}} W^{\frac{2 N}{N-2}}+\int_{\Omega_{\varepsilon}} W^{\frac{2 N}{N-2}}\left(W^{\varepsilon}-1\right) \\
& =\int_{\Omega_{\varepsilon}}(U-\hat{\varphi})^{\frac{2 N}{N-2}}+\varepsilon \int_{\Omega_{\varepsilon}}(U-\hat{\varphi})^{\frac{2 N}{N-2}} \ln (U-\hat{\varphi})+O\left(\varepsilon^{2}|\ln \varepsilon|\right) \\
& =\int_{\Omega_{\varepsilon}} U^{\frac{2 N}{N-2}}-\frac{2 N}{N-2} \int_{\Omega_{\varepsilon}} U^{\frac{N+2}{N-2}} \hat{\varphi}+\varepsilon \int_{\Omega_{\varepsilon}}(U-\hat{\varphi})^{\frac{2 N}{N-2}} \ln (U-\hat{\varphi})+O\left(\varepsilon^{2}|\ln \varepsilon|\right) .
\end{aligned}
$$


The validity of this expansion can be verified by Lebesgue's Dominated Convergence Theorem and the fact that $|W-U| \leqslant C \varepsilon|\ln \varepsilon|^{n} U_{\frac{1}{\Lambda}, a}^{\frac{N-3}{N-2}}$ (see the first inequality in (A.8) and similar arguments in Section 5 of [34]). Note also that

$$
\int_{\Omega_{\varepsilon}}(U-\hat{\varphi})^{\frac{2 N}{N-2}} \ln (U-\hat{\varphi})=-\frac{N-2}{2} \ln \Lambda \int_{\mathbb{R}_{+}^{N}} U_{1,0}^{\frac{2 N}{N-2}}+\int_{\mathbb{R}_{+}^{N}} U_{1,0}^{\frac{2 N}{N-2}} \ln U_{1,0}+O\left(\varepsilon^{1-\tau}\right) .
$$

Then, according to the definition (3.28) of $J_{\varepsilon}$ and $\alpha_{N}=N(N-2)$

$$
\begin{aligned}
J_{\varepsilon}(W)= & \left((N-2)+\frac{(N-2)^{3}}{4 N} \varepsilon\right) \int_{\Omega_{\varepsilon}} U^{\frac{2 N}{N-2}}+\frac{N(N-2)}{2} \int_{\Omega_{\varepsilon}} U^{\frac{N+2}{N-2}} \hat{\varphi} \\
& +\frac{(N-2)^{3}}{4} \varepsilon \ln \Lambda \int_{\mathbb{R}_{+}^{N}} U_{1,0}^{\frac{2 N}{N-2}}-\varepsilon \frac{(N-2)^{2}}{2} \int_{\mathbb{R}_{+}^{N}} U_{1,0}^{\frac{2 N}{N-2}} \ln U_{1,0}+O\left(\varepsilon^{2-\tau}\right)
\end{aligned}
$$

noticing (see estimates below), that $\int_{\Omega_{\varepsilon}} U^{\frac{2 N}{N-2}}=O(1)$ and $\int_{\Omega_{\varepsilon}} U^{\frac{N+2}{N-2}} \hat{\varphi}=O\left(\varepsilon^{1-\tau}\right)$. We observe that

$$
\begin{aligned}
\int_{\Omega_{\varepsilon}} U^{\frac{2 N}{N-2}} & =\int_{\mathbb{R}_{+}^{N}} U_{\frac{1}{\Lambda}, 0}^{\frac{2 N}{N-2}}\left(y^{\prime}, y_{N}+\frac{\rho\left(\varepsilon y^{\prime}\right)}{\varepsilon}\right)+O\left(\varepsilon^{2-\tau}\right) \\
& =\int_{\mathbb{R}_{+}^{N}} U_{\frac{1}{\Lambda}, 0}^{\frac{2 N}{N-2}}\left(y^{\prime}, y_{N}\right)+\int_{\mathbb{R}_{+}^{N}} \frac{\partial U_{\frac{1}{\Lambda}, 0}^{\frac{2 N}{N-2}}}{\partial y_{N}}\left(y^{\prime}, y_{N}\right)\left(\frac{\rho\left(\varepsilon y^{\prime}\right)}{\varepsilon}\right)+O\left(\varepsilon^{2-\tau}\right)
\end{aligned}
$$

whence

$$
\int_{\Omega_{\varepsilon}} U^{\frac{2 N}{N-2}}=\int_{\mathbb{R}_{+}^{N}} U_{1,0}^{\frac{2 N}{N-2}}-\frac{1}{2} \Lambda \varepsilon H(a) \int_{\partial \mathbb{R}_{+}^{N}} U_{1,0}^{\frac{2 N}{N-2}}|y|^{2} \mathrm{~d} y+O\left(\varepsilon^{2-\tau}\right) .
$$

On the other hand, in view of the expansion of $\varphi_{\Lambda, a, \mu, \varepsilon}$ in Lemma A.1, we also have

$$
\begin{aligned}
\alpha_{N} \int_{\Omega_{\varepsilon}} U^{\frac{N+2}{N-2}} \hat{\varphi}_{\Lambda, a, \mu, \varepsilon} & =\Lambda \varepsilon \alpha_{N} \int_{\Omega_{\varepsilon}} U_{1,0}^{\frac{N+2}{N-2}} \varphi_{0}+O\left(\varepsilon^{2-\tau}\right)=\Lambda \varepsilon \alpha_{N} \int_{\mathbb{R}_{+}^{N}} U_{1,0}^{\frac{N+2}{N-2}} \varphi_{0}+O\left(\varepsilon^{2-\tau}\right) \\
& =\Lambda \varepsilon \int_{\mathbb{R}_{+}^{N}}\left(-\Delta U_{1,0} \varphi_{0}+U_{1,0} \Delta \varphi_{0}\right)+O\left(\varepsilon^{2-\tau}\right)=\Lambda \varepsilon \int_{\partial \mathbb{R}_{+}^{N}}\left(-\frac{\partial \varphi_{0}}{\partial y_{N}} U_{1,0}\right)+O\left(\varepsilon^{2-\tau}\right) \\
& =-\Lambda \varepsilon \frac{N-2}{2} \sum_{j=1}^{N-1} k_{j} \int_{\partial \mathbb{R}_{+}^{N}} U_{1,0} \frac{y_{j}^{2}}{\left(1+|y|^{2}\right)^{\frac{N}{2}}}+O\left(\varepsilon^{2-\tau}\right) .
\end{aligned}
$$

Therefore

$$
\alpha_{N} \int_{\Omega_{\varepsilon}} U^{\frac{N+2}{N-2}} \hat{\varphi}_{\Lambda, a, \mu, \varepsilon}=-\Lambda \varepsilon \frac{N-2}{2} H(a) \int_{\partial \mathbb{R}_{+}^{N}} \frac{|y|^{2}}{\left(1+|y|^{2}\right)^{N-1}}+O\left(\varepsilon^{2-\tau}\right) .
$$


Substituting (A.14) and (A.15) into (A.13), we obtain

$$
J_{\varepsilon}(W)=A-B^{*} \Lambda \varepsilon H(a)+\frac{(N-2)^{2}}{4} A \varepsilon \ln \Lambda+\varepsilon\left(\frac{(N-2)^{2}}{4 N} A+C\right)+O\left(\varepsilon^{2-\tau}\right)
$$

where $A, C$ are given in (A.11) and

$$
B^{*}=\frac{N-2}{2} \int_{\partial \mathbb{R}_{+}^{N}} U_{1,0}^{\frac{2 N}{N-2}}|y|^{2}+\frac{N-2}{4} \int_{\partial \mathbb{R}_{+}^{N}} \frac{|y|^{2}}{\left(1+|y|^{2}\right)^{N-1}} .
$$

To make the proof of Proposition A.1 complete, it only remains to show that $B^{*}=B$ defined by (A.12). In fact, it is easily seen that

$$
\int_{\partial \mathbb{R}_{+}^{N}} U_{1,0}^{\frac{2 N}{N-2}}|y|^{2}=\omega_{N-2} \int_{0}^{\infty} \frac{r^{N}}{\left(1+r^{2}\right)^{N}} \mathrm{~d} r=\frac{N-3}{2(N-1)} \omega_{N-2} \int_{0}^{\infty} \frac{r^{N}}{\left(1+r^{2}\right)^{N-1}} \mathrm{~d} r
$$

where $\omega_{N-2}$ is the area of the unit sphere in $R^{N-1}$. The last equality follows from simple integration by parts. Then, we can rewrite $B^{*}$ as

$$
B^{*}=B=\frac{(N-2)^{2}}{N-3} \int_{\partial \mathbb{R}_{+}^{N}} U_{1,0}^{\frac{2 N}{N-2}}|y|^{2} .
$$

The expansions for the derivatives of $J_{\varepsilon}$ are obtained exactly in the same way.

\section{A.3. Proof of Lemma 3.2}

We prove (3.6) first. Through scaling, we may assume that $\varepsilon=1$. Let $G(x, y)$ be the Green's function satisfying

$$
-\Delta G(x, y)+\mu G(x, y)=\delta_{y} \quad \text { in } \Omega, \quad \frac{\partial G(x, y)}{\partial n}=0 \quad \text { on } \partial \Omega .
$$

Then we have for $x \in \Omega$,

$$
u(x)=\int_{\Omega} G(x, y) f(y) \mathrm{d} y .
$$

So it is enough to show that there exists a constant $C$, independent of $x$ and $y$, such that

$$
|G(x, y)| \leqslant \frac{C}{|x-y|^{N-2}} .
$$

To this end, we decompose $G$ in two parts:

$$
G(x, y)=K(|x-y|)+H(x, y)
$$

where $K(|x-y|)$ is the singular part of $G$ and $H(x, y)$ is the regular part of $G$. Certainly we have $|K(|x-y|)| \leqslant$ $\frac{C}{|x-y|^{N-2}}$. It remains to show that

$$
|H(x, y)| \leqslant \frac{C}{|x-y|^{N-2}} .
$$

Note that, if $d(x, \partial \Omega)>d_{0}>0$ or $d(y, \partial \Omega)>d_{0}>0$, then $|H(x, y)| \leqslant C$ and hence (A.16) also holds. So we just need to estimate $H(x, y)$ for $d(x, \partial \Omega)$ and $d(y, \partial \Omega)$ small. Let $y \in \Omega$ be such that $d=d(y, \partial \Omega)$ is small. So there exists a unique point $\bar{y} \in \partial \Omega$ such that $d=|y-\bar{y}|$. Without loss of generality, we may assume $\bar{y}=0$ and the 
outer normal at $\bar{y}$ is pointing toward $x_{N}$-direction. Let $y^{*}$ be the reflection point $y^{*}=(0, \ldots, 0,-d)$ and consider the following auxiliary function

$$
H^{*}(x, y)=K\left(\left|x-y^{*}\right|\right) .
$$

Then $H^{*}$ satisfies $\Delta H^{*}-\mu H^{*}=0$ in $\Omega$ and on $\partial \Omega$

$$
\frac{\partial}{\partial n}\left(H^{*}(x, y)\right)=-\frac{\partial}{\partial n}(K(|x-y|))+O\left(\frac{1}{d^{N-3}}\right) .
$$

Hence we derive that

$$
H(x, y)=-H^{*}(x, y)+O\left(\frac{1}{d^{N-3}}\right)
$$

which proves (A.16) for $x, y \in \Omega$. This implies that for $x \in \Omega$

$$
|u(x)| \leqslant C \int_{\Omega} \frac{1}{|x-y|^{N-2}}|f(y)| \mathrm{d} y .
$$

If $x \in \partial \Omega$, we consider a sequence of points $x_{i} \in \Omega, x_{i} \rightarrow x \in \partial \Omega$ and take the limit in (A.17). Lebesgue's Dominated Convergence Theorem applies and (3.6) is proved.

We turn now to the proof of (3.7). By Lemma 3.1, we have

$$
\|u\|_{L_{\beta}^{t}\left(\Omega_{\varepsilon}\right)} \leqslant C\|f\|_{L_{\beta+2}^{t}\left(\Omega_{\varepsilon}\right)}
$$

hence

$$
\left\|\varepsilon^{2} u\right\|_{L_{\beta+2}^{t}\left(\Omega_{\varepsilon}\right)} \leqslant C\|u\|_{L_{\beta}^{t}\left(\Omega_{\varepsilon}\right)} \leqslant C\|f\|_{L_{\beta+2}^{t}\left(\Omega_{\varepsilon}\right)} .
$$

By a usual transformation and extension (as done in Step 2 of Proof of Theorem 2.1 in [30]) and interpolation, one can show that

$$
\|u\|_{W_{\beta}^{2, t}\left(B_{\delta / \varepsilon}(\xi)\right)} \leqslant C\left\|\varepsilon^{2} u\right\|_{L_{\beta+2}^{t}\left(\Omega_{\varepsilon}\right)}+C\|f\|_{L_{\beta+2}^{t}\left(\Omega_{\varepsilon}\right)} \leqslant C\|f\|_{L_{\beta+2}^{t}\left(\Omega_{\varepsilon}\right)},
$$

where $\delta$ is a small fixed constant. Next we take a cut-off function $\chi(x)$ such that $\chi(x)=1$ for $|x| \leqslant \frac{\delta}{2}$ and $\chi(x)=0$ for $|x|>\delta$, and we consider the function

$$
u^{1}(x)=u(y)(1-\chi(\varepsilon y-\xi))
$$

which satisfies

$$
-\Delta_{x} u^{1}+\mu \varepsilon^{2} u^{1}=2 \varepsilon \nabla_{y} u \cdot \nabla_{x} \chi+\varepsilon^{2} u \Delta_{x} \chi+f(1-\chi)
$$

in $\tilde{\Omega}=\Omega \backslash\{|x-a|<\delta\}$. Applying the elliptic regularity theory, we obtain

$$
\left\|u^{1}\right\|_{W^{2, t}(\tilde{\Omega})} \leqslant C\left\|2 \varepsilon \nabla_{y} u \nabla_{x} \chi+\varepsilon^{2} u \Delta_{x} \chi+f(1-\chi)\right\|_{L^{t}(\tilde{\Omega})}
$$

whence, taking account of (A.18)

$$
\left\|u^{1}\right\|_{W_{\beta}^{2, t}\left(\Omega_{\varepsilon} \backslash B_{\frac{\delta}{\varepsilon}}(\xi)\right)} \leqslant C\|f\|_{L^{t}(\tilde{\Omega})}+C \varepsilon^{\beta+2}\|f\|_{L_{\beta+2}^{t}\left(\Omega_{\varepsilon}\right)} .
$$

Combining (A.18) and (A.19), we obtain (3.7). 


\section{References}

[1] Adimurthi, G. Mancini, The Neumann problem for elliptic equations with critical nonlinearity, "A tribute in honour of G. Prodi”, Scuola Norm. Sup. Pisa (1991) 9-25.

[2] Adimurthi, G. Mancini, Geometry and topology of the boundary in the critical Neumann problem, J. Reine Angew. Math. 456 (1994) $1-18$.

[3] Adimurthi, F. Pacella, S.L. Yadava, Interaction between the geometry of the boundary and positive solutions of a semilinear Neumann problem with critical nonlinearity, J. Funct. Anal. 113 (1993) 318-350.

[4] A. Bahri, Critical Points at Infinity in Some Variational Problems, Pitman Res. Notes Math. Ser., vol. 182, Longman, 1989.

[5] P. Bates, G. Fusco, Equilibria with many nuclei for the Cahn-Hilliard equation, J. Differential Equations 160 (2000) $283-356$.

[6] L. Caffarelli, B. Gidas, J. Spruck, Asymptotic symmetry and local behavior of semilinear elliptic equations with critical Sobolev growth, Comm. Pure Appl. Math. 42 (1989) 271-297.

[7] G. Cerami, J. Wei, Multiplicity of multiple interior peaks solutions for some singularly perturbed Neumann problems, Intern. Math. Res. Notes 12 (1998) 601-626.

[8] E.N. Dancer, S. Yan, Multipeak solutions for a singularly perturbed Neumann problem, Pacific J. Math. 189 (1999) $241-262$.

[9] M. Del Pino, P. Felmer, M. Musso, Two-bubble solutions in the super-critical Bahri-Coron's problem, Calc. Var. Partial Differential Equations 16 (2003) 113-145.

[10] M. Grossi, A. Pistoia, On the effect of critical points of distance function in superlinear elliptic problems, Adv. Differential Equations 5 (2000) 1397-1420.

[11] M. Grossi, A. Pistoia, J. Wei, Existence of multipeak solutions for a semilinear elliptic problem via nonsmooth critical point theory, Calc. Var. Partial Differential Equations 11 (2000) 143-175.

[12] A. Gierer, H. Meinhardt, A theory of biological pattern formation, Kybernetik (Berlin) 12 (1972) 30-39.

[13] C. Gui, Multi-peak solutions for a semilinear Neumann problem, Duke Math. J. 84 (1996) 739-769.

[14] C. Gui, C.S. Lin, Estimates for boundary-bubbling solutions to an elliptic Neumann problem, J. Reine Angew. Math. 546 (2002) $201-235$.

[15] C. Gui, J. Wei, Multiple interior peak solutions for some singularly perturbed Neumann problems, J. Differential Equations 158 (1999) $1-27$.

[16] C. Gui, J. Wei, On the existence of arbitrary number of bubbles for some semilinear elliptic equations with critical Sobolev exponent, in press.

[17] C. Gui, J. Wei, On multiple mixed interior and boundary peak solutions for some singularly perturbed Neumann problems, Canad. J. Math. 52 (2000) 522-538.

[18] C. Gui, J. Wei, M. Winter, Multiple boundary peak solutions for some singularly perturbed Neumann problems, Ann. Inst. H. Poincaré Anal. Non Linéaire 17 (2000) 47-82.

[19] S. Khenissy, O. Rey, A criterion for existence of solutions to the supercritical Bahri-Coron's problem, Houston J. Math. 30 (2004) 587613.

[20] M. Kowalczyk, Multiple spike layers in the shadow Gierer-Meinhardt system: existence of equilibria and quasi-invariant manifold, Duke Math. J. 98 (1999) 59-111.

[21] Y. Li, W.-M. Ni, On conformal scalar curvature equation in $R^{n}$, Duke Math. J. 57 (1988) 895-924.

[22] Y.Y. Li, On a singularly perturbed equation with Neumann boundary condition, Comm. Partial Differential Equations 23 (1998) $487-545$.

[23] C.S. Lin, W.M. Ni, On the Diffusion Coefficient of a Semilinear Neumann Problem, Lecture Notes in Math., vol. 1340, Springer, New York, 1986.

[24] C.S. Lin, W.N. Ni, I. Takagi, Large amplitude stationary solutions to a chemotaxis system, J. Differential Equations 72 (1988) 1-27.

[25] R. McOwen, The behavior of the Laplacian on weighted Sobolev spaces, Comm. Pure Appl. Math. 32 (1979) $783-795$.

[26] S. Maier-Paape, K. Schmitt, Z.Q. Wang, On Neumann problems for semilinear elliptic equations with critical nonlinearity: existence and symmetry of multi-peaked solutions, Comm. Partial Differential Equations 22 (1997) 1493-1527.

[27] W.-M. Ni, Diffusion, cross-diffusion, and their spike-layer steady states, Notices Amer. Math. Soc. 45 (1998) 9-18.

[28] W.N. Ni, X.B. Pan, I. Takagi, Singular behavior of least-energy solutions of a semi-linear Neumann problem involving critical Sobolev exponents, Duke Math. J. 67 (1992) 1-20.

[29] W.N. Ni, I. Takagi, On the shape of least-energy solutions to a semi-linear problem Neumann problem, Comm. Pure Appl. Math. 44 (1991) 819-851.

[30] W.M. Ni, I. Takagi, Locating the peaks of least-energy solutions to a semi-linear Neumann problem, Duke Math. J. 70 (1993) $247-281$.

[31] O. Rey, The role of the Green's function in a nonlinear elliptic problem involving the critical Sobolev exponent, J. Funct. Anal. 89 (1990) $1-52$.

[32] O. Rey, An elliptic Neumann problem with critical nonlinearity in three dimensional domains, Comm. Contemp. Math. 1 (1999) $405-449$.

[33] O. Rey, The question of interior blow-up points for an elliptic Neumann problem: the critical case, J. Math. Pures Appl. 81 (2002) $655-696$.

[34] O. Rey, J. Wei, Blow-up solutions for an elliptic Neumann problem with sub- or supercritical nonlinearity, I: $N=3$, J. Funct. Anal., in press.

[35] X.J. Wang, Neumann problem of semilinear elliptic equations involving critical Sobolev exponents, J. Differential Equations 93 (1991) 283-310. 
[36] Z.Q. Wang, The effect of domain geometry on the number of positive solutions of Neumann problems with critical exponents, Differential Integral Equations 8 (1995) 1533-1554.

[37] Z.Q. Wang, High energy and multi-peaked solutions for a nonlinear Neumann problem with critical exponent, Proc. Roy. Soc. Edinburgh Sect. A 125 (1995) 1003-1029.

[38] Z.Q. Wang, Construction of multi-peaked solution for a nonlinear Neumann problem with critical exponent, J. Nonlinear Anal. 27 (1996) 1281-1306.

[39] X. Wang, J. Wei, On the equation $\Delta u+K(x), u^{\frac{n+2}{n-2} \pm \epsilon^{2}}=0$ in $R^{n}$, Rend. Circ. Mat. Palermo 2 (1995) 365-400.

[40] J. Wei, On the interior spike layer solutions of singularly perturbed semilinear Neumann problems, Tohoku Math. J. 50 (1998) $159-178$.

[41] J. Wei, X. Xu, Uniqueness and a priori estimates for some nonlinear elliptic Neumann equations in $\mathbb{R}^{3}$, Pacific J. Math., in press.

[42] J. Wei, M. Winter, Stationary solutions for the Cahn-Hilliard equation, Ann. Inst. H. Poincaré, Anal. Non Linéaire 15 (1998) $459-482$.

[43] S. Yan, On the number of interior multipeak solutions for singularly perturbed Neumann problems, Topol. Methods Nonlinear Anal. 12 (1998) 61-78.

[44] M. Zhu, Uniqueness results through a priori estimates, I. A three dimensional Neumann problem, J. Differential Equations 154 (1999) 284-317. 